\title{
Emergent Lorentz symmetry and the Unruh effect in a Lorentzian fermionic tensor network
}

\author{
Zhi Yang, ${ }^{2, \dagger}$ Arpan Bhattacharyya, ${ }^{5, ‡}$ Long Cheng, ${ }^{4,2, *}$ Ling-Yan Hung, ${ }^{1,2,3, \S}$ and Sirui Ning ${ }^{6, \|}$ \\ ${ }^{1}$ State Key Laboratory of Surface Physics and Department of Physics, Fudan University, \\ 220 Handan Road, 200433 Shanghai, People's Republic of China \\ ${ }^{2}$ Department of Physics and Center for Field Theory and Particle Physics, Fudan University, \\ 220 Handan Road, 200433 Shanghai, People's Republic of China \\ ${ }^{3}$ Collaborative Innovation Center of Advanced Microstructures, Nanjing University, Nanjing 210093, \\ People's Republic of China \\ ${ }^{4}$ Department of Physics, Zhejiang Sci-Tech University, Hangzhou 310018, People's Republic of China \\ ${ }^{5}$ Yukawa Institute for Theoretical Physics (YITP), Kyoto University, \\ Kitashirakawa Oiwakecho, Sakyo-ku, Kyoto 606-8502, Japan \\ ${ }^{6}$ School of the Gifted Young, University of Science and Technology of China, \\ Hefei, 230026 Anhui, People's Republic of China
}

(Received 25 November 2018; published 9 April 2019)

\begin{abstract}
In this paper we attempt to understand Lorentzian tensor networks, as a preparation for constructing tensor networks that can describe more exotic backgrounds such as black holes. To define notions of reference frames and switching of reference frames on a tensor network, we will borrow ideas from the algebraic quantum field theory literature. With these definitions, we construct simple examples of Lorentzian tensor networks, based on Gaussian models of fermions, and solve the spectrum for a choice of "inertial frame." In particular, the tensor network can be viewed as a periodically driven Floquet system that bypasses the "doubling problem" and gives rise to fermions with exactly linear dispersion relations. We will find that a boost operator connecting different inertial frames and notions of "Rindler observers" can be defined and that important physics in Lorentz invariant quantum field theory, such as the Unruh effect, can be captured by such a skeleton of spacetime. We find interesting subtleties when the same approach is directly applied to bosons - the operator algebra contains commutators that take the wrong sign—resembling bosons behind horizons.
\end{abstract}

DOI: 10.1103/PhysRevD.99.086007

\section{INTRODUCTION}

Interesting aspects of the AdS/CFT, such as reconstructing black hole physics from CFT data, are crucial for understanding quantum gravity. There has been an increasing amount of evidence showing that the AdS/CFT can be understood in terms of a tensor network since the possibility was first pointed out in [1]. There are interesting toy constructions of tensor networks attempting to capture the physics, particularly of black holes. For example, it is noted that a black hole should probably behave like a region of

\footnotetext{
* Corresponding author. lcheng@fudan.edu.cn

zyang14@fudan.edu.cn

*bhattacharyya.arpan@yahoo.com

§elektron.janethung@gmail.com

"cmicheal@mail.ustc.edu.cn
}

Published by the American Physical Society under the terms of the Creative Commons Attribution 4.0 International license. Further distribution of this work must maintain attribution to the author(s) and the published article's title, journal citation, and DOI. Funded by SCOAP . particularly high bond dimension in a tensor network [2,3]. These constructions, however, are (mostly) based on static spacetimes, and the tensor network considered essentially describes the Euclidean geometry of some time slice. The complete description of black holes, particularly if we were to address questions such as unitarity, is a time-dependent question. Interesting physics is associated with the black hole horizon, and it would thus require notions of null surfaces, and therefore also notions of spacelike and timelike separations in a tensor network construction. Therefore, to gain an understanding of gravitational dynamics via tensor networks requires us at ground zero to define how spacelike/timelike separations are described in a tensor network in the first place.

There is some recent progress based on a random tensor network that also attempts to describe a covariant version of the AdS/CFT [4]. The current paper has a relatively modest goal, taking a step toward understanding what it means to represent spacetime on a tensor network. In this paper we would like to explore how one should recover crucial features of a Lorentzian spacetime and understand the causal structures and their implications for different spacetime observers. 
To that end, we also need to set up the problem and define notions such as observers on a tensor network.

Our paper is divided into two parts. First, we would like to put together the language that has been developed in the tensor network/error correcting code/bulk-reconstruction literature with notions developed in algebraic quantum field theory (AQFT). In Sec. II, we review just enough basics so that in Sec. III, we define notions of causality, Cauchy surfaces, and frames of references in a tensor network.

Then in Sec. IV, using these definitions, we construct explicit models. We start with a quadratic fermionic model and demonstrate, at least in some limits very explicitly, that notions such as Lorentz transformation and the Unruh effect can be described to very good approximation in the tensor network. In fact, the tensor network evolution is effectively turning the system into a periodically driven Floquet system that could give rise to a linear dispersion relation free of the "doubling problem." (For a review of the problem, see, for example, [5].) These fermions can have exactly linear dispersion relations with emergent Lorentz invariance that becomes evident in the computation of the correlation functions and anticommutation relations. We will also see that light cones depend on the explicit choice of the tensors furbishing the tensor network.

This hopefully lays out some basic features of tensor networks describing Lorentzian spacetime and serves as preparation as we move on to a covariant construction of more general interesting spacetimes based on the tensor networks.

\section{AXIOMS OF AQFT}

In this section we give a very brief summary of the basic axioms defining an AQFT, listing all the ingredients that are going to have a natural realization in a tensor network. Our discussion is heavily based on [6], which gives a relatively gentle review of the subject. We also find [7] a concise and physical exposition of the subject. The Kaag-Hastler axioms are motivated by incorporating locality and causality into an operator algebra that in turn defines a QFT.

The ingredients involved are therefore spacetime manifold $\mathcal{M}$ on the one hand and some operator algebra $\mathcal{A}$ on the other. There are various conditions imposed on $\mathcal{M}$. Of course traditionally, the discussion is based on smooth manifolds. We will list all the ingredients and discuss which can make direct contact with the tensor network. First, we need some constraints on the set of spacetimes concerned.

(1) $\mathcal{M}$ as a topological space is Hausdorff, connected and paracompact.

(2) $\mathcal{M}$ has a pseudo-Riemannian metric $g$. This defines a causal structure. For a smooth curve $\gamma(t)$ in $\mathcal{M}$, it can either be spacelike if $g(\dot{\gamma}, \dot{\gamma})<0$ or causal (timelike or null-like) if $g(\dot{\gamma}, \dot{\gamma}) \geq 0$.

(3) $\mathcal{M}$ is globally hyperbolic such that it does not contain closed causal curves and for any two points $x$ and $y, J_{+}(x) \cap J_{-}(y)$ is compact. Here $J_{+}(x)$ denotes the collection of points that are in the "future" of $x$ and that can be connected to $x$ by a future directed causal curve. $J_{-}(x)$ corresponds to the causal past of $x$.

(4) Having a causal structure also means that we can define an order relation $x<y$, i.e., $x$ precedes $y$ if there exists a future directing causal curve connecting $x$ to $y$. This relation can be generalized to subsets in $\mathcal{M}$. Consider two sets $O_{1}$ and $O_{2}$. They are spacelike separated if they cannot be connected by a casual curve-if for all $x \in \bar{O}_{1}, J_{ \pm}(x)$ has an empty intersection with $\mathrm{O}_{2}$.

(5) There exist foliations by Cauchy surfaces, (locally) diffeomorphic to $\Sigma \times R$.

(6) There exist "admissible embeddings" $\chi: \mathcal{M} \rightarrow \mathcal{N}$ for globally hyperbolic spacetimes $\mathcal{M}, \mathcal{N}$, such that the map preserves the metric, orientations, and causal structure.

(7) Collection $B$ of bounded subsets of $\mathcal{M}$ forms a directed set. There exists a reflexive and transitive binary relation: for a pair $O_{1}, O_{2} \in B, \exists O: O_{1} \subseteq O$ and $O_{2} \subseteq O$.

These spacetimes form a "category" $L O C$ - a category of locally hyperbolic, oriented, and time-oriented spacetimes.

With such a spacetime as a starting point, the AQFT is a rule of assigning an operator algebra to hyperbolic spacetimes. Mathematically, an AQFT is a functor that maps between two categories: $L O C$ on one hand and $O B S$ - the category of unital $C^{*}$-algebra corresponding to the algebra of operators of physical observables - on the other.

In QFT, it is taken that observables form a $C^{*}$-algebra. For completeness, we provide the definition of $C^{*}$-algebra below. A $C^{*}$-algebra $A$ comes with a norm $\|x\|$ and a map * that takes $A \rightarrow A$. For $x \in A$, where $A$

$$
\begin{gathered}
x^{* *}=\left(x^{*}\right)^{*}=x, \\
(x+y)^{*}=x^{*}+y^{*}, \\
(\lambda x)^{*}=\bar{\lambda} x^{*} \\
\left\|x^{*} x\right\|=\|x\|\left\|x^{*}\right\| .
\end{gathered}
$$

The " $C^{*}$ " stands for "(norm-)closed." The norm provides the algebra with a topological structure. Let us make contact with quantum mechanics. Consider $H$ to be a complex Hilbert space with inner product denoted $\langle\cdot, \cdot\rangle$. The collection of bounded linear operators on $H$, denoted by $B(H)$, is a $C^{*}$-algebra. The linear structure is clear. The product is by composition of operators. The $*$ operation is the adjoint; for any operator $a$ on $H$, its adjoint is defined by the equation $\left\langle a^{*} \zeta, \eta\right\rangle=\langle\zeta, a \eta\rangle$, for all $\zeta$ and $\eta$ in $H$. Finally, the norm is given by $\|a\|=\sup \{\|a \zeta\| \zeta \in H$, $|\zeta| \leq 1\}$, for any $a$ in $B(H)$. 
The axioms constraining the AQFT functor $\mathcal{U}$ are as follows: $\mathcal{U}$ assigns to each bounded subset $O \in B$ a $C^{*}$-algebra $\mathcal{U}(O)$. The algebra of spacetime $\mathcal{M}$ is defined as the inductive limit

$$
\mathcal{U}(\mathcal{M})=\overline{\cup_{O} \mathcal{U}(O)} .
$$

(This can be understood as a generalization of a direct sum.) Since this is a map from a directed set $B$ to another set, it forms a net of $C^{*}$-algebras. For any subset $\mathcal{N} \subset \mathcal{M}$, $\mathcal{U}(\mathcal{N})$ is generated by $\mathcal{U}(O)$ where $O \subset \mathcal{N}$, which satisfy the following list of axioms,

(1) Isotony. For $O \subset \tilde{O}, \mathcal{U}(O) \subset \mathcal{U}(\tilde{O})$. This expresses the fact that the operator algebra abides by the notion of $\subset$ in spacetime $\mathcal{M}$.

(2) Locality (Einstein causality). When $O_{1}$ and $O_{2}$ are spacelike separated as defined above, $[A, B]=0$, for all $A \in \mathcal{U}\left(O_{1}\right)$ and $B \in \mathcal{U}\left(O_{2}\right)$.

(3) Time slice axiom. The solvability of the initial value problem is translated into a requirement of the operator algebra. The algebra $\mathcal{U}(N)$ is isomorphic to $\mathcal{U}(\mathcal{M})$ for any causally convex neighbourhood of a Cauchy surface $\Sigma$. Note that a causally convex neighbourhood $N$ is one in which no causal curve inside $\mathcal{M}$ meets $N$ in a disconnected set.

(4) In a generally curved spacetime, it is more suitable to invoke local charts and they can be understood as the admissible maps $\chi$ discussed above. For each admissible embedding $\chi: N \rightarrow M$, there is an injective homomorphism $\alpha_{\chi}: \mathcal{U}(N) \rightarrow \mathcal{U}(M)$. If $\chi_{1}: M \rightarrow N$ and $\chi_{2}: N \rightarrow L$, then we have

$$
\alpha_{\chi_{2} \circ \chi_{2}}=\alpha_{\chi_{2}} \circ \alpha_{\chi_{1}} ;
$$

i.e., altogether, the assignment of algebra via $\mathcal{U}$ has to be compatible with the structures of embeddings leading to maps between algebras. That makes an AQFT a covariant functor between $L O C$ and $O B S$. In terms of these embedding maps, the Einstein causality constraint can be phrased as follows: if $\chi_{1}\left(M_{1}\right) \subset M$ and $\chi_{2}\left(M_{2}\right) \subset M$ are causally disconnected, then

$$
\left[\alpha_{\chi_{1}}\left(\mathcal{U}\left(M_{1}\right)\right), \alpha_{\chi_{2}}\left(\mathcal{U}\left(M_{2}\right)\right)\right]=0 .
$$

Similarly, the time-slice axiom can be restated if we introduce an embedding map $\chi: N \rightarrow M$, where $N$ is the causally convex neighborhood of a Cauchy surface, and we have $\alpha_{\chi}$ as an isomorphism.

(5) The above isomorphism describes general covariance. If we only have global isometries, then the statement is instead restricted to the existence of an isomorphism $\alpha_{L}$ that maps $\alpha_{L}: \mathcal{U}(O) \rightarrow \mathcal{U}(L O)$, where $L O$ is the region $O$ transformed via some isometry $L$ (such as Poincaré symmetry in Minkowski spacetime).

(6) Time evolution between any two Cauchy surfaces: given two Cauchy surfaces $\Sigma_{1,2}$, the neighborhood
$N_{i}$ of each can be associated with an embedding map $\chi_{i}: N_{i} \rightarrow M$ such that $\alpha_{\chi_{i}}$ is an algebra isomorphism from $\mathcal{U}\left(N_{i}\right) \rightarrow \mathcal{U}(M)$. One can obtain an algebra isomorphism between $\alpha_{1 \rightarrow 2}: \mathcal{U}\left(N_{1}\right) \rightarrow \mathcal{U}\left(N_{2}\right)$, where

$$
\alpha_{1 \rightarrow 2}=\alpha_{\chi_{2}}^{-1} \circ \alpha_{\chi_{1}} .
$$

\section{CONSTRUCTING A CAUSAL SPACETIME USING A TENSOR NETWORK}

We would like to build a causal spacetime using a tensor network by associating building components of a tensor network to ingredients in an AQFT discussed above.

A tensor network is a collection of tensors contracted with each other, with each tensor represented as a vertex, and shared contracted indices between tensors represented by an edge connecting these vertices. Therefore in this simplest version, the tensor network associates a graph to a collection of tensors.

\section{A. Topological space}

A graph $F$ can be viewed as a topological space naturally endowed with 0 simplices (vertices) and 1-simplices (edges). This topological space is the arena that plays the role of the spacetime manifold $M$. Clearly there are more structures to spacetimes than such a skeleton, although not everything has an immediate analogue in the tensor network.

Notwithstanding, a graph is a set of vertices and edges, and as such, the notion of $O_{i} \subset O_{j}$, where $O_{i}$ and $O_{j}$ are subgraphs of $F$, is well defined. Therefore, this is also a directed set.

\section{B. Hilbert space and local operator algebra}

Each edge is associated to a contracted index of a tensor. Therefore each edge can be naturally associated with a Hilbert space. (Perhaps practically finite dimensional.) Operators acting on this Hilbert space would thus form a $C^{*}$-algebra, as discussed in the previous section. Associating an operator algebra to links does not constitute $\mathcal{U}(O)$. These operators are related to each other. Such relations will be laid out below.

\section{Causal structure: Timelike vs spacelike separation}

The graph does not generically possess any causal structure. The associated tensor network without any causal structure might well be associated to a Euclidean version of spacetime. To describe Lorentzian signature, the graph needs to acquire a causal structure.

One way of incorporating causal structure in the tensor network is based on local unitaries.

Consider the special case in which each tensor $T$ has an even number of legs, each with the same bond dimension. 
We can split the indices into two equal groups $g_{1,2}$. If $T_{\alpha_{i} \in g_{1}}^{\beta_{j} \in g_{2}}$ is a unitary from $\alpha_{i}$ to $\beta_{j}$, then we would consider the vertices connected via these edges to be causally connected. This thus assigns a causal ordering in the graph.

Such orderings can only be made consistent with each other, however, as follows. Consider two indices $\alpha_{1} \in g_{1}$ and $\beta_{1} \in g_{2}$, where we have assigned the ordering $g_{1} \prec g_{2}$. If there exists another split into two groups $g_{3}$ and $g_{4}$ such that $\alpha_{1}$ and $\beta_{1}$ belong to $g_{3}$, then at least one index in $g_{1}$ must now belong to $g_{4}$ and at least another one in $g_{2}$ now belongs to $g_{3}$. In such a case, requiring either $g_{3} \prec g_{4}$ or vice versa would be inconsistent with $g_{1} \prec g_{2}$. Therefore a consistent causal ordering assignment can be made only if there is a unique splitting $g_{1}, g_{2}$ such that $T$ is unitary. In which case, edges within the same group can be considered spacelike separated. For each set of unitary evolution, we are defining a set of observers, or a frame.

For $T$ being perfect tensors (a $2 \mathrm{n}$-index tensor $T_{a 1 a 2 \ldots a 2 n}$ is a perfect tensor if, for any bipartition of its indices into a set $A$ and complementary set $A^{c}$ with $|A| \leq\left|A^{c}\right|$, T is proportional to an isometric tensor from $A$ to $A^{c}$ [2]) for example, then every pair of indices is timelike separated, and yet there is no ordering agreed upon by all frames. In other words, there is no consistent assignment of a causal precedence in this case.

Such an assignment is local. The above assignment makes it natural to include arrows in the edges to denote causal precedence. We can put ingoing arrows among edges in $g_{1}$ and outgoing arrows in edges in $g_{2}$. As a unitary matrix, the number of arrows is conserved across each vertex.

For a global assignment of causal structure, one needs to pay special attention to how edges are contracted with each other. With the arrow assignment, a global causal structure would follow when these directed tensors are assembled together, where each outgoing edge proceeds to become an ingoing edge in the other vertex it connects to.

Global assignment of a causal structure thus requires that the graph $F$ is orientable. The requirement of the absence of closed timelike curves becomes the requirement that the graph is a directed acyclic graph, which is a finite directed graph with no directed cycles. The causal structure is borne out by the building block being local unitaries. This is similar to the consideration in the causal-set approach to quantum gravity. (This is a huge subject in its own right which is impossible to review here. We refer interested readers to one of the original papers [8,9] and references therein for more recent discussions.) In the current paper, the emergent causality in a tensor network is ultimately a measurement problem of commutators based on Einstein locality. They are not predetermined at the level of the graph even though the structure of the graph could preclude various causal structures.

This point of view will be emphasized again in Sec. III C 4 below. In the context of the tensor networks it is not clear whether graphs that are transitively closed/completegraphs where there exists an edge connecting any two causally related points - play any special role.

To summarize, it appears that the conservation of ingoing and outgoing legs on a directed acyclic graph matched with local tensors with a unique "unitary direction" has replaced the notion of global hyperbolicity.

\section{Operator pushing and local unitaries}

The local unitaries $T$ define isomorphisms between operator algebras. Operators from ingoing legs are related to operators in the outgoing legs by conjugation (or equivalently what is called "operator pushing," for example, in [2]). An immediate consequence is that as we push an operator starting from a Cauchy slice across $T$ 's, we sweep out a light cone, either forward or backward in time, a feature already observed, for example, in the context of multiscale entanglement renormalization ansatz (MERA) tensor networks [10]. This ensures that information cannot flow faster than the speed of light, which is captured by the Einstein causality condition below.

\section{Operator algebra assigned to a connected subgraph $O$}

Now consider $O$ to be a connected subgraph of $F$. Then we can pick out a set of edges all mutually spacelike separated. The operator algebra $\mathcal{U}(O)$ associated to this region $O$ can be defined as the operator algebra on the maximal set $S$ of mutually spacelike separated edges. Operators acting on any other legs can be pulled back to operators acting on $S$ via local unitaries. Or in other words, we will include in this operator algebra only those that can be pulled back to $S$. This defines $\mathcal{U}(O)$ that is generated by the operator algebra on $S$. To make subsequent discussion simple, when we discuss these subregions $O$, they should carry the structure of a causal diamond-the boundary of $O$ should intersect $\partial S$. The boundary is thus separated into two pieces by $\partial S$. Each piece is related to $S$ by a unitary map.

Observation on the tensor network: If $\mathrm{O}_{1} \subset \mathrm{O}_{2}$, then $S_{1} \subset S_{2}$ and thus $\mathcal{U}\left(O_{1}\right) \subset \mathcal{U}\left(O_{2}\right)$. The set of algebra $\{\mathcal{U}(O)\}$ also forms a net of $\left(C^{*}\right)$ algebra.

\section{Cauchy surface and the time slice axiom}

For an acyclic directed graph, it admits a slicing which is a set of edges, such that no two are related causally and that

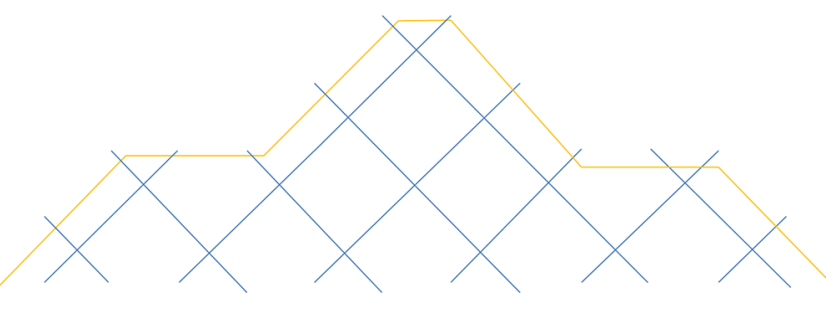

FIG. 1. A typical Cauchy surface in the network. 


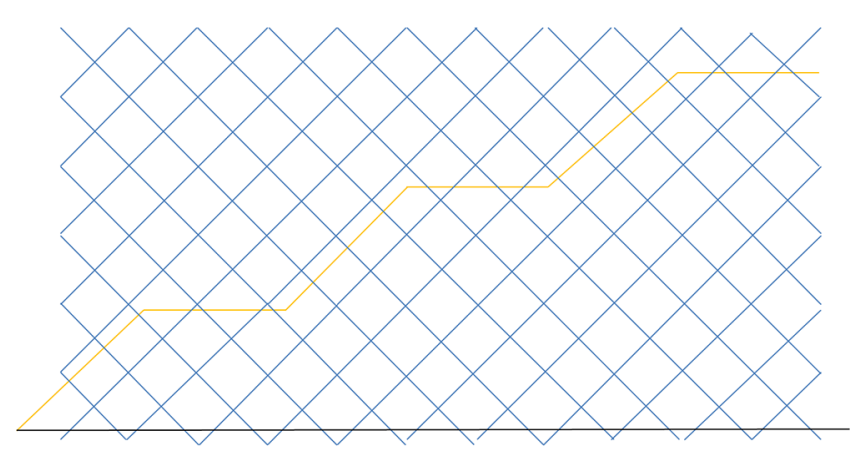

FIG. 2. The set of networks between the two Cauchy surfaces (the horizontal Cauchy surface marked black and the ladderlike Cauchy surface marked yellow) is a unitary matrix, and we define that as the discrete approximation of a Lorentz transformation.

the surface does not have a boundary. Such a surface can generically be chosen as a Cauchy slice $\Sigma$. A typical Cauchy surface on the network is illustrated in Fig. 1.

A neighborhood $N$ of a Cauchy surface $\Sigma$ can now be defined as picking up new edges connected to edges on the Cauchy surface by the tensors $T$. Since individual $T$ are all local unitaries, this defines an algebra $\mathcal{U}(N)$ which is isomorphic to the algebra generated by the $C^{*}$-algebra on $\Sigma$ via the unitary maps $T$. This is also isomorphic to the algebra $\mathcal{U}(F)$, which is isomorphic to the $C^{*}$-algebra at any Cauchy surface $\Sigma$.

Algebras on different Cauchy surfaces are related also by isomorphism. The isomorphic map $\alpha_{1 \rightarrow 2}: \mathcal{U}\left(\Sigma_{1}\right) \rightarrow \mathcal{U}\left(\Sigma_{2}\right)$ is now provided by the sets of $T$ contained between two Cauchy surfaces $\Sigma_{1,2}$ and this defines a unitary evolution.

That any two choices of Cauchy surfaces can be related by a unitary map is probably equivalent to the Stone-von Neumann theorem in finite dimensional Hilbert spaces. (See, for example, [7] for an explanation of the Stone-von Neumann theorem.) Figure 2 illustrates a unitary transformation between the horizontal surface and the slanted ladderlike surface. As we are going to see, in a homogeneous network where every tensor is the same, this can be interpreted as an approximate Lorentz transformation.

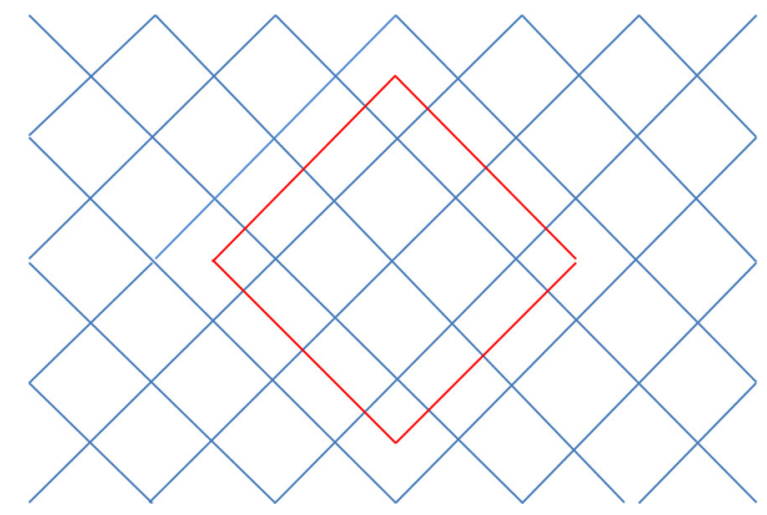

FIG. 3. Causal diamond.
A set of observers defining a "frame" is characterized by an ordered set of Cauchy surfaces, and these Cauchy surfaces are related by unitary transformations. If there is time translation invariance, it corresponds to the fact that the unitary matrices that map one Cauchy surface to the next remain unchanged.

\section{Einstein locality}

To make an actual comparison between the two types of operator algebra $\mathcal{U}\left(O_{1,2}\right)$ assigned to two different connected subgraphs $O_{1,2}$, we embed them into $\mathcal{U}(\Sigma)$ for any choice of Cauchy surface $\Sigma$, which defines an embedding map $\alpha_{1,2 \rightarrow F}: \mathcal{U}\left(O_{1,2}\right) \rightarrow \mathcal{U}(F)$, since $\mathcal{U}(F)$ is itself isomorphic to $\mathcal{U}(\Sigma)$.

With that, we can define Einstein locality between two spacelike separated regions $O_{1,2}$ using $\alpha_{1,2 \rightarrow F}$ :

$$
\left[\alpha_{1}\left(\mathcal{U}\left(O_{1}\right)\right), \alpha_{2}\left(\mathcal{U}\left(O_{2}\right)\right)\right]=0 .
$$

As noted above, these maps $\alpha_{i}$ of operators are basically operators pushing across tensors $T$ along unitary directions. The above commutation relations can be phrased equivalently as follows. Consider a Cauchy surface $\Sigma_{1}$ containing $S_{1}$. Now pull the operators in $O_{2}$ to $\Sigma_{1}$, which we denote by the map $\alpha_{2 \rightarrow \Sigma_{1}}$. If $\alpha_{2 \rightarrow \Sigma_{1}}\left(\mathcal{U}\left(O_{2}\right)\right) \subset \mathcal{U}\left(P_{1}\right)$, where $P_{1} \subset \Sigma_{1}$ and $S_{1} \cap P_{1}=0$, then (9) is satisfied.

Thus far, the tensor network falls short of being a "functor" mapping the category of graphs to $O B S$. It is not obvious what physical data go into defining such a functor and how it can be compared with a quantum field theory. ${ }^{1}$

Note that to explicitly compute these commutators, we first construct Cauchy surfaces that contain each of these locations and then perform operator pushing of one operator in one of the Cauchy surfaces to the other surface. The precise choice of the Cauchy surface is immaterial since the tensors only act locally, but the existence of which is crucial. That would essentially rule out much potential confusion over whether two points are in fact connected causally.

\section{Isotony}

Isotony can be defined as follows in the tensor network. We will restrict our attention to subregions in spacetime describable by causal diamonds. The algebra attached to a causal diamond can be described as follows. We locate the spacelike surface and the intersection of its causal future and causal past to define a causal diamond. The operator algebra

\footnotetext{
${ }^{1}$ When defining a quantum field theory, a standard procedure is to define a Lagrangian for some given set of fields. The program based on category theory defines a quantum field theory without using a Lagrangian. For a CFT, one needs a set of primaries, their conformal dimensions and their OPEs to completely specify the CFT. It is not completely clear to the authors what the full set of data is that is needed to specify a generic QFT in this language. A tensor network probably has more data than are necessary.
} 
associated to this region of space is defined as the operator algebra defined along the maximal spacelike surface inside the causal diamond Fig. 3. Any spacelike surfaces are thus related to the maximal spacelike surface by isomorphisms.

Now if a causal diamond is inside another causal diamond, it is always possible to map the algebra on the spacelike surface in the smaller diamond to the algebra on the maximal spacelike surface in the larger diamond. The operator algebra is sure to be a subalgebra of the algebra defined on the larger causal diamond. Therefore, an algebra net is naturally recovered for causal diamonds.

Note that for a spacelike region $A$ with a causal diamond $D(A)$, it follows that the entanglement entropy only depends on $D(A)$ but not on the specific maximal spacelike surface $A$ chosen inside $D(A)$. This is because all these maximal spacelike surfaces inside $D(A)$ are all related by local unitaries which preserve entanglement entropy.

\section{ILLUSTRATION BASED ON FREE FERMIONS}

In this section we would like to illustrate some of these ideas using a simple model. We would like to construct a unitary evolution that is explicitly expressed as a tensor network of local unitaries. Then we would like to construct notions familiar in a continuous spacetime in the present context and show that they can be approximated to some extent.

\section{A. Tensor network evolution}

First of all, we have to define a model that is inspired by the free fermions but whose time evolution takes the form of a tensor network built from local unitaries. In fact, breaking up a generic unitary evolution into a product of local unitaries is frequently employed in numerical simulations or actual experiments on quantum simulations $[11,12]$. The Lieb-Robinson bound is also based on such an approximation [13]. Here, however, we will take the viewpoint that the tensor network defines the model.

Our tensor network is constructed as follows. Consider a set of fermion creation and annihilation operators $a_{i}, a_{i}^{\dagger}$, where $i$ denotes the link where the fermion is located. These operators satisfy the usual anticommutator:

$$
\left\{a_{i}, a_{j}^{\dagger}\right\}=\delta_{i, j} .
$$

To construct the simplest example of a unitary evolution, we consider the quadratic Hamiltonian,

$H=\sum_{i=-2 L+1}^{2 L-1} h_{i, i+1}, \quad h_{i, i+1}=\alpha\left(a_{i}^{\dagger} a_{i+1}+a_{i} a_{i+1}^{\dagger}\right)$,

where $2 L$ is the size of the spin chain. We label the vertices by $-L+\frac{1}{2},-L+\frac{3}{2}, \ldots, L-\frac{3}{2}, L-\frac{1}{2}$. Thus, the links run from $-2 L+1$ to $2 L-1$. We have already taken lattice spacing $a=1$ here.
To ensure that $H$ is Hermitian, we take

$$
\alpha=\frac{\tilde{\alpha}}{2 i}
$$

for some real $\tilde{\alpha}$.

The time evolution operator $U(\Delta t)$ over the smallest unit of time $\Delta t$ is given by

$$
U(\Delta t)=\left(\prod_{i} U_{2 i, 2 i+1}\right)\left(\prod_{j} U_{2 j-1,2 j}\right),
$$

where

$$
U_{i, i+1}=\exp \left(i \Delta t h_{i, i+1}\right) .
$$

We have

$U a_{2 x} U^{-1}=c^{2} a_{2 x}+c s a_{2 x-1}-c s a_{2 x+1}+s^{2} a_{2 x+2}$

and

$U a_{2 x+1} U^{-1}=c^{2} a_{2 x+1}-c s a_{2 x+2}+c s a_{2 x}+s^{2} a_{2 x-1}$,

where

$$
c=\cos (i \alpha \Delta t), \quad s=\sin (i \alpha \Delta t),
$$

and $x$ is the label of the "site" on a given Cauchy surfacethe vertex to which the links are connected. For the sake of convenience in later (numerical) computations of correlation functions, we have labeled these vertices by halfintegers $x$ and the links $i$ by integers. We therefore have $i=2 x+1$ labeling "even" links and $i=2 x$ labeling "odd" links. The labelling is illustrated also in Fig. 4. Time evolution is given by repeated application of $U$. There is

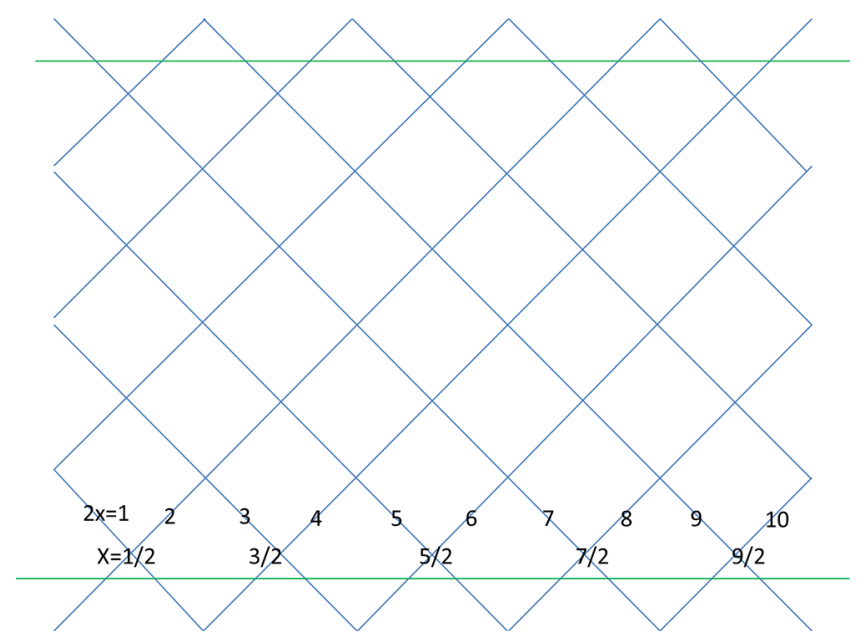

FIG. 4. The collection of horizontal Cauchy surfaces, defining a particular set of "inertial observers." We note that we adopt a rather odd labeling for convenience with numerics and take "site" numbers as half-integers $x$, while links are labeled by integers $2 x, 2 x+1$. 
thus a time translation invariance over $t \rightarrow t+2 \Delta t$. The simplest set of Cauchy surfaces are those that are "horizontal." We will take these collection of "Cauchy slices" to define an inertial frame. The tensor network and the labeling are illustrated in Fig. 4.

The network is invariant under translation by two links $i \rightarrow i+2$. Therefore based on translation invariance, we expect that the eigenmodes in this inertial frame should be given by plane waves. We will solve for the spectrum in this frame in the following.

\section{Spectra of the model}

From the translation symmetry of the network, we expect the eigenoperators to be expressible as

$$
a_{p}=\sum_{x=-L+\frac{1}{2}}^{L-\frac{1}{2}}\left(f_{2 x} a_{2 x}+g_{2 x+1} a_{2 x+1}\right),
$$

for some coefficients $f_{2 x}(p)$ and $g_{2 x+1}(p)$ given by

$$
f_{2 x}=q_{1} e^{i p x}, \quad g_{2 x+1}=q_{2} e^{i p x},
$$

where $q_{1,2}$ are independent of the site number $x$. They are to be determined by the following eigenvalue equations. The momentum is given by

$$
p=n \frac{2 \pi}{2 L},
$$

where $n$ are integers satisfying $-L \leq n \leq L-1$. In the limit $L \rightarrow+\infty$, we can take $-\pi<p<\pi$. The eigenvalue equation is obtained by demanding

$$
U a_{p} U^{-1}=\lambda a_{p}
$$

This implies

$$
\begin{aligned}
& f_{2 x} c^{2}+g_{2 x+1} c s+f_{2 x-2} s^{2}-g_{2 x-1} c s=\lambda f_{2 x}, \\
& g_{2 x+1} c^{2}+g_{2 x+3} s^{2}+f_{2 x+2} s c-f_{2 x} s c=\lambda g_{2 x+1} .
\end{aligned}
$$

For each given $p$, it gives two eigenmodes with eigenvalues

$\lambda_{ \pm}=\left(c^{2}+s^{2} \cos (p)\right) \pm i \sqrt{\left(1-\left(c^{2}+s^{2} \cos (p)\right)^{2}\right)}$.

We note that the eigenvalues are even in the momentum $p$. In the limit $c \rightarrow 0$ the eigenvalue approaches

$$
\lim _{c \rightarrow 0} \lambda_{ \pm}(p)=\exp ( \pm i|p|)
$$

which appears as if its energy is linearly dependent on $p$. This recovers the dispersion of a relativistic free massless fermion without the doubling problem. In the other limit where $c \rightarrow 1$, we have $\lambda(p)$ approaching a constant. The model becomes nondispersive. Therefore while the graph on which the model is defined remains unchanged, the effective causal structure of the system changes dramatically as the unit of time $\Delta t$ in the model is varied.

For $\lambda_{-}$, the corresponding eigenmodes are given by

$$
\frac{q_{2}^{-}}{q_{1}^{-}} \equiv \frac{\alpha_{2}}{\alpha_{1}}=\frac{e^{\frac{i p}{2}}\left(s \sin (p)-\sqrt{(1-\cos (p))\left(2 c^{2}+s^{2} \cos (p)+s^{2}\right)}\right)}{2 c \sin \left(\frac{p}{2}\right)} .
$$

Then for $\lambda_{+}$the eigenmodes are

$$
\frac{q_{2}^{+}}{q_{1}^{+}} \equiv \frac{\beta_{2}}{\beta_{1}}=\frac{e^{\frac{i p}{2}}\left(s \sin (p)+\sqrt{(1-\cos (p))\left(2 c^{2}+s^{2} \cos (p)+s^{2}\right)}\right)}{2 c \sin \left(\frac{p}{2}\right)} .
$$

In the above expressions, when we take the limit $c \rightarrow 0$, the case with positive $p$ and negative $p$ should be treated separately. We will take this limit in the subsequent analysis.

We can then define the operators corresponding to creation and annihilation of these eigenmodes:

$$
\begin{aligned}
& a_{p}=\mathcal{N} \sum_{x=-L+\frac{1}{2}}^{L-\frac{1}{2}} e^{i p x}\left(\alpha_{1} a_{2 x}+\alpha_{2} a_{2 x+1}\right), \\
& b_{p}^{\dagger}=\mathcal{N} \sum_{x=-L+\frac{1}{2}}^{L-\frac{1}{2}} e^{i p x}\left(\beta_{1} a_{2 x}+\beta_{2} a_{2 x+1}\right) . \\
& \mathcal{N}=\frac{1}{\sqrt{2 L}} .
\end{aligned}
$$

We check that

$$
\left\{a_{p}, a_{p}^{\dagger}\right\}=1
$$

which implies

$$
\alpha_{1} \alpha_{1}^{*}+\alpha_{2} \alpha_{2}^{*}=1,
$$

and similarly for $b_{p}$. This gives

$$
\beta_{1} \beta_{1}^{*}+\beta_{2} \beta_{2}^{*}=1 .
$$

On the other hand, Eq. (19) implies

$$
\alpha_{1} \beta_{1}^{*}+\alpha_{2} \beta_{2}^{*}=0,
$$

which means that 


$$
\left\{a_{p}, b_{p}^{\dagger}\right\}=0,
$$

so $b_{p}^{\dagger}$ and $a_{p}$ behave as creation and annihilation operators, respectively. We can now define the fermion field as

$a_{2 x}(x, t)=\mathcal{N} \sum_{n=-L}^{L-1} e^{-i p x}\left(\frac{a_{p} e^{-i|\chi| t}}{\alpha_{1}\left(1-\frac{\alpha_{2} \beta_{1}}{\alpha_{1} \beta_{2}}\right)}+\frac{b_{p}^{\dagger} e^{i|\chi| t}}{\beta_{1}\left(1-\frac{\beta_{2} \alpha_{1}}{\beta_{1} \alpha_{2}}\right)}\right)$,

where

$$
\cos (\chi)=c^{2}+s^{2} \cos (p) .
$$

Similarly,

$$
a_{2 x+1}(x, t)=\mathcal{N} \sum_{n=-L}^{L-1} e^{-i p x}\left(\frac{a_{p} e^{-i|\chi| t}}{\alpha_{2}\left(1-\frac{\alpha_{1} \beta_{2}}{\alpha_{2} \beta_{1}}\right)}+\frac{b_{p}^{\dagger} e^{i|\chi| t}}{\beta_{2}\left(1-\frac{\beta_{1} \alpha_{2}}{\beta_{2} \alpha_{1}}\right)}\right) .
$$

We would like to define the vacuum state as

$$
a_{p}|0\rangle=0, \quad b_{p}|0\rangle=0 .
$$

In a discrete spacetime, the notion of a "ground state" is clearly ill defined. If the smallest unit of time is $\Delta t$, then the energy is identified with a period, i.e., $E \sim E+2 \pi / \Delta t$. However, mimicking the continuous situation we can look for a special state that corresponds to a "separating vector"- - reference to distinguish the "positive/negative" energy modes. Requiring that in the $p \rightarrow 0$ limit the "low energy modes" so defined by this state should recover our usual notion of low energy states (i.e., energy should decrease with decreasing momentum), this special state would be a natural "vacuum" state. This is essentially the procedure that we have followed above. We note that while a discrete evolution in Lorentzian signature does not identify a ground state without ambiguity, a Euclidean

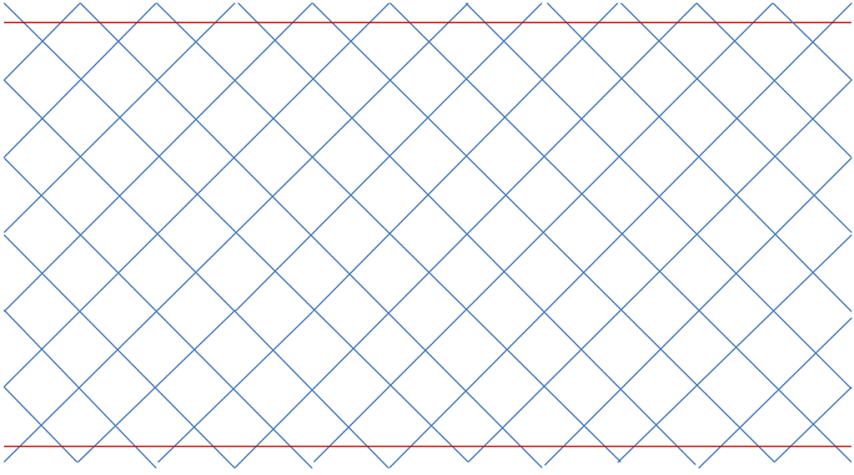

(a) partition function would continue to project to a unique (unless otherwise degenerate) ground state. One wonders how such an analytic continuation can be defined if time were discrete. The folklore that a quantum model in $d$ dimensions necessarily originates from a classical model in $d+1$ dimensions is by no means obvious when time becomes discrete. As we have seen above, variation of $\Delta t$ while keeping the Hamiltonian unchanged could lead to utterly different dispersion relations and subsequently causal structures. It appears that at least in the case of integrable models, however, an analytic continuation between Lorentzian and Euclidean signatures remains well defined. This is discussed in our accompanying paper [14].

\section{B. Lorentz transformation}

Since the system has a well-defined causal structure, it is very tempting to define the notion of boost, i.e., the set of (unitary) transformations between different observers. As already explained in the previous section, an observer is defined as a collection of Cauchy surfaces. The unitary transformation connecting observers is generically given by the set of tensors sandwiched between the respective Cauchy surfaces. In general, such a transformation does not preserve the ground state. For a general set of observers, there is not even any notion of time translation invariance, such that energy is completely ill defined. For our model, there are different observers that enjoy some degree of time translation invariance. This is illustrated in Fig. 5.

If in the special case that these different observers agree (perhaps only approximately) upon the notion of "positive energy" modes, it means that the vacuum state remains invariant under the corresponding transformation between these observers. These observers that (approximately) share the same ground state would be the closest analogue we have for "inertial observers" in a continuous Lorentz invariant spacetime.

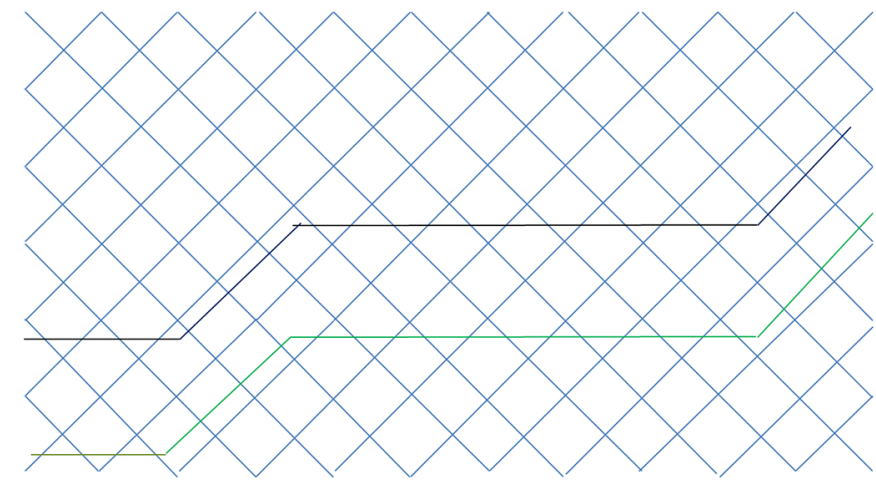

(b)

FIG. 5. This diagram illustrates two independent sets of inertial observers (a) and (b), marked by sets of Cauchy surfaces with different colors. The tensors sandwiched between each pair of Cauchy surfaces of the same color correspond to the corresponding inertial time evolution between the pair. 
In the current tensor network we are working with, there are natural families of Cauchy surfaces corresponding to inertial observers, inspired by results in continuous spacetime. Each family of Cauchy surfaces is slanted surfaces with some given slope. We note, however, that in a discrete spacetime, these slanted surfaces with given slopes are more accurately speaking ladders. For a given slope, the corresponding Cauchy "ladder" is not unique, and we understand them as slightly different approximations of these inertial observers which only become indistinguishable in the long-wavelength limit.

A boost transformation that relates these different observers is constructed below.

\section{Approximating the boost operator}

Consider the simplest scenario. There is a natural set of inertial observers with flat Cauchy surfaces. We consider another set of inertial observers over ladder surfaces. We would like to construct the unitary boost operator that transforms the ladder into the flat surface. The most natural guess would correspond to the set of local unitaries sandwiched between the two Cauchy surfaces. However, after connecting the Cauchy surfaces, in general one can do further transformations that preserve the target Cauchy surface, such as translations on the surface. We therefore allow the boost operator to take the general form, corresponding to one that first translates along the time direction according to the sandwiched unitaries, which could be, depending on the situation at hand, followed by a set of translations along the target Cauchy surface. This is the same strategy taken in [15] in the Euclidean version when constructing transformations mapping between different (Cauchy) surfaces.

We can compute the effect these transformations have on our modes. To make the discussions most transparent and the similarity of our model with the continuous case obvious, let us first consider the limit $c \rightarrow 0$, in which case we recall that the dispersion relations (20) reduce to a linear one. That is directly analogous to the discussion of the free fermions in the $c \rightarrow 0$ limit in the main text.

We note that in the limit $c \rightarrow 0$, the fermion evolution (19) behaves like a (fermionic) swap, in which even links are translated to the left by two steps $2 x+1 \rightarrow 2 x-1$ and odd links are being translated to the right also by two steps $2 x \rightarrow 2 x+2$. This suggests that the fermionic modes turn into a pair of chiral fermions propagating in opposite directions. In fact, one can directly check the expressions (22) and (23) to confirm that modes with positive momenta have positive (negative) energies for the odd (even) links. This is recapped below. We list the eigenoperators explicitly as $c \rightarrow 0$. We note that, as $c \rightarrow 0$, the expression for the operator approaches a rather singular limit, where special care has to be taken when taking limits of (22) and (23), the correlation functions are relatively straightforward to treat. Nonetheless, it is possible to show that the modes reduce to

$$
\begin{aligned}
& a_{p<0}=-\mathcal{N} \sum_{x} e^{i p x} e^{i \frac{p}{2}} a_{2 x+1}, \\
& a_{p<0}^{\dagger}=-\mathcal{N} \sum_{x} e^{-i p x} e^{-i \frac{p}{2}} a_{2 x+1}^{\dagger}, \\
& b_{p \geq 0}=\mathcal{N} \sum_{x} e^{-i p x} e^{-i \frac{p}{2}} a_{2 x+1}^{\dagger}, \\
& b_{p \geq 0}^{\dagger}=\mathcal{N} \sum_{x} e^{i p x} e^{i \frac{p}{2}} a_{2 x+1},
\end{aligned}
$$

and then similarly

$$
\begin{aligned}
& a_{p \geq 0}=\mathcal{N} \sum_{x} e^{i p x} a_{2 x}, \quad a_{p \geq 0}^{\dagger}=\mathcal{N} \sum_{x} e^{-i p x} a_{2 x}^{\dagger}, \\
& b_{p<0}=-\mathcal{N} \sum_{x} e^{-i p x} a_{2 x}^{\dagger}, \quad b_{p<0}^{\dagger}=-\mathcal{N} \sum_{x} e^{i p x} a_{2 x} .
\end{aligned}
$$

The vacuum continues to be the one that is annihilated by $a_{p}$ and $b_{p}$.

The inverse transform would give

$$
\begin{aligned}
a_{2 x} & =\mathcal{N} \sum_{p} e^{-i p x}\left(a_{p \geq 0}-b_{p<0}^{\dagger}\right), \\
a_{2 x+1} & =\mathcal{N} \sum_{p} e^{-i p x} e^{-i \frac{p}{2}}\left(-a_{p<0}+b_{p \geq 0}^{\dagger}\right) .
\end{aligned}
$$

In this case, we can inspect the effect the boost operator has on the operators $a_{p}$ and $b_{p}$.

The boost operator that we will illustrate in detail is shown in Fig. 6.

The effect of the ladder operator $\hat{B}$ has on $a_{i}$ is as follows. One can see that in Fig. 6 for $i>0$, i.e., $x=\frac{1}{2}, \frac{3}{2}, \cdots$,

$$
\begin{array}{r}
i=2 x, \quad \Delta i=w\left(x+\frac{1}{2}\right), \quad \text { where } w=2 \\
i=2 x+1, \quad \Delta x=-\frac{w}{3} x-f(x),
\end{array}
$$

where

$$
f(x)= \begin{cases}\frac{2}{3}, & x-\frac{1}{2}=0(\bmod 3) \\ 1, & x-\frac{1}{2}=1(\bmod 3) \\ \frac{1}{3}, & x-\frac{1}{2}=2(\bmod 3)\end{cases}
$$

For $i=0$, the operator $a_{0}$ is invariant under the transformation of the ladder operator $\hat{B}$. For $i<0$,

$$
\begin{array}{r}
i=2 x, \quad\left(x=-\frac{1}{2},-\frac{3}{2}, \cdots\right) \quad \Delta i=w\left(x-\frac{1}{2}\right), \text { where } w=2 \\
i=2 x+1,\left(x=-\frac{3}{2},-\frac{5}{2}, \cdots\right) \quad \Delta x=-\frac{w}{3} x-g(x),
\end{array}
$$




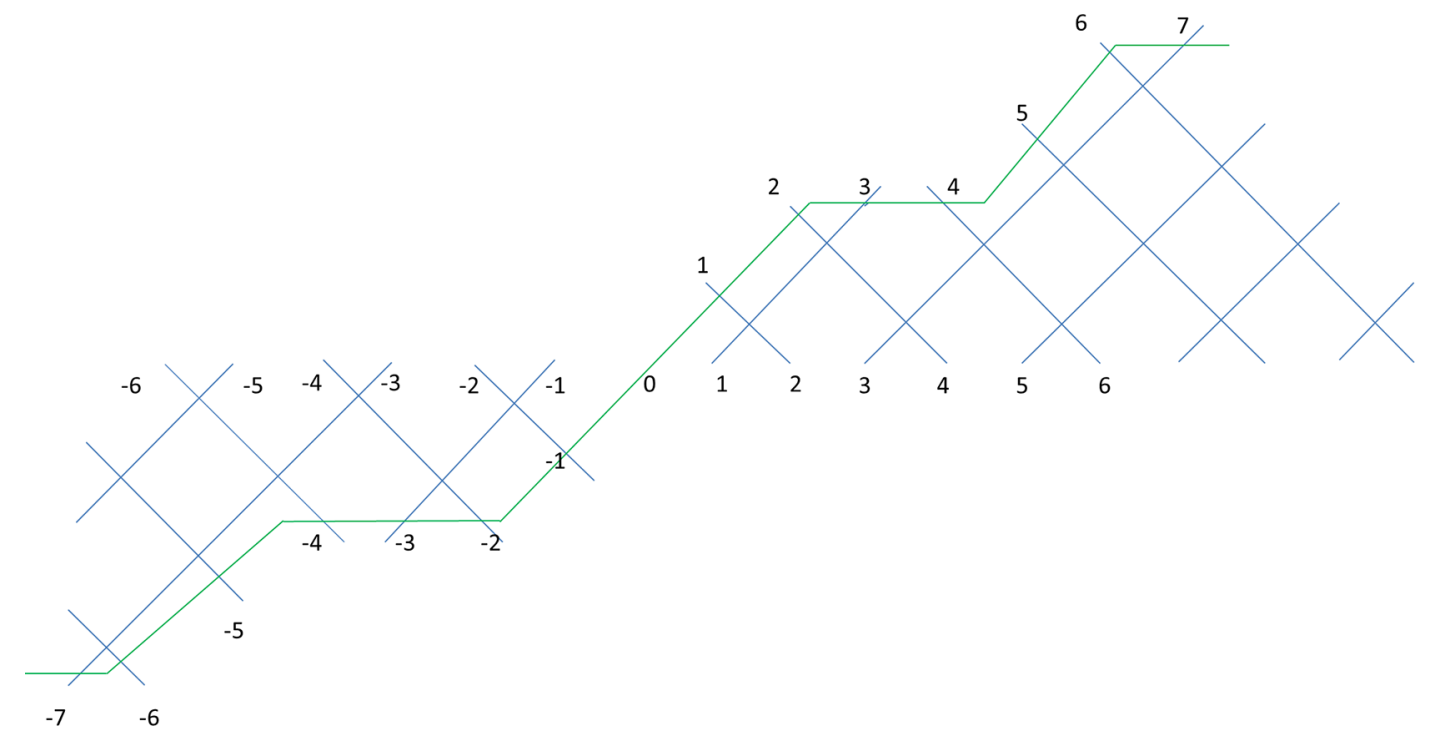

FIG. 6. The explicit form of a particular boost operator.

where

$$
g(x)=\left\{\begin{array}{cl}
-\frac{1}{3}, & x-\frac{1}{2}=0(\bmod 3) \\
0, & x-\frac{1}{2}=1(\bmod 3) . \\
\frac{1}{3}, & x-\frac{1}{2}=2(\bmod 3)
\end{array}\right.
$$

In general, we could consider also the effect of a linkdependent translation that takes

$$
i \rightarrow M i,
$$

for some (odd) integer $M$ which would preserve the current Cauchy surface. ${ }^{2}$ But in the current illustration, let us do without further deformation of the Cauchy surface, which does not appear to alter the physics of the final result.

Given the above expressions, one readily obtains the effect the boost operator has on the modes. For positive links, using (37), we obtain

$$
\begin{aligned}
\hat{B} a_{2 x} \hat{B}^{-1} & =a_{2((1+w / 2) x+w / 4)} \\
& =\mathcal{N} \int_{-\pi}^{\pi} \frac{d p}{2 \pi} e^{-i p((1+w / 2) x+w / 4)}\left(a_{p \geq 0}-b_{p<0}^{\dagger}\right), \\
\hat{B} a_{2 x+1} \hat{B}^{-1} & =a_{\left(2-\frac{w}{3}\right) x+1-f(x) .}
\end{aligned}
$$

Let us emphasize here that the apparent shift by $w / 4$ above is an unfortunate result of our notation which takes $x$ to be half-integers. The reader should be able to see from Fig. 6 that the odd links particularly are simply scaled, i.e., $1 \rightarrow$ $3,3 \rightarrow 7$ etc., with the steps scaling linearly with the link number. The even links behave similarly with a slight complication of some internal structure in the rescaling which is explained in Eq. (37) and easily recovered also from the same figure. Since our transformation only makes sense in the thermodynamic limit where the momentum also approaches a continuous limit, we replace the summation over $p$ by an integral over the first Brillouin zone. Note that the lattice spacing is just $a=1$ here. For negative links, using (39), we obtain

$$
\begin{aligned}
\hat{B} a_{2 x} \hat{B}^{-1} & =a_{2((1+w / 2) x-w / 4)}, \\
\hat{B} a_{2 x+1} \hat{B}^{-1} & =a_{\left(2-\frac{w}{3}\right) x+1-g(x)} .
\end{aligned}
$$

To obtain the effect it has on individual $a_{p \geq 0}$, we take another inverse Fourier transform to get

$$
\begin{aligned}
\hat{B} a_{q \geq 0} \hat{B}^{-1}= & \mathcal{N} \sum_{x>0} e^{i q x} a_{2((1+w / 2) x+w / 4)}+\mathcal{N} \sum_{x<0} e^{i q x} a_{2((1+w / 2) x-w / 4)}=\mathcal{N} \sum_{x>0} \int_{-\pi}^{\pi} \frac{d p}{2 \pi} e^{i(q x-p((1+w / 2) x+w / 4))}\left(a_{p \geq 0}-b_{p<0}^{\dagger}\right) \\
& +\mathcal{N} \sum_{x<0} \int_{-\pi}^{\pi} \frac{d p}{2 \pi} e^{i(q x-p((1+w / 2) x-w / 4))}\left(a_{p \geq 0}-b_{p<0}^{\dagger}\right) .
\end{aligned}
$$

\footnotetext{
${ }^{2} M$ being odd ensures that even links and odd links remain decoupled for simple solutions. Also we are taking the infinite size limit of the lattice for the transformation to take the same form everywhere.
} 
The above modes live only on the odd links. Similar expressions can be obtained for $b_{p<0}$ and $a_{p<0}$ which depend on the even links, although one can expect from (37) that the result would be less clean.

The interesting observation is that in the thermodynamic limit where the total number of sites approaches infinity, the sum over sites gives us a delta function $\delta(q-p(1+\omega / 2))$. For small $p>0$ and that $(1+\omega / 2) p \leq \pi$,

$$
\hat{B}(\Lambda) a_{p} \hat{B}^{-1}(\Lambda)=a_{\Lambda^{-1} p}, \quad \Lambda=e^{\eta},
$$

where we have denoted $\Lambda=(1+w / 2)$. We note that in the current example at hand $\Lambda=2$. We thus recover the expected transformation of the modes under a Lorentz boost. Importantly, the boost operator does not exchange the annihilation operator for creation operation in this long-wavelength limit. For $\Lambda p>\pi$, one would have mapped a positive energy mode to a negative energy one. In other words, under the operation of our guess of a "boost," it (almost up to some lattice effect which disappears as $L \rightarrow \infty$ and $|p|$ sufficiently small, i.e., a continuous limit) preserves the positive and negative energy modes, which is a necessary ingredient of a Lorentz transformation in a Lorentz invariant theory that preserves the ground state.

Due to our choice of the form of the ladders, it does not treat the $p>0$ and $p<0$ modes in a completely symmetric manner, because the ladder breaks the symmetry between even and odd links. However, we associate such deviations with lattice effects.

\section{Spectra of the boost operator}

In the previous subsection, we made a crude guess of the form of the boost operator. To proceed with understanding the Unruh effect, we would like to solve for the spectrum of the boost operator, and obtain eigenmodes in the $x<0$ and $x>0$ halves of the lattice. These modes should behave like eigenmodes observed by a Rindler (accelerated) observer.

Since the boost operator does not act on the link at $i=0$, it essentially breaks up the space into two halves; the eigenmodes on the two halves of the lattice decouple. Therefore, the form of the eigenmodes can be considered separately on the right and left halves of the lattice.

We will continue to work with $c=0$ to illustrate the point, where even and odd links remain decoupled.

We begin with solving for eigenoperators on the right side of the lattice. We first write down an ansatz for the eigenmodes. Since

$$
A_{\kappa}^{R}=\sum_{x>0} \psi_{\kappa}(x) a_{2 x}
$$

then we require that

$$
\hat{B} A_{\kappa}^{R} \hat{B}^{-1}=\eta_{R}(\kappa) A_{\kappa}^{R}
$$

This gives

$$
\sum_{x>0} \psi_{\kappa}(x) a_{2((1+w / 2) x+w / 4)}=\eta_{R}(\kappa) \sum_{x>0} \psi_{\kappa}(x) a_{2 x} .
$$

In momentum space, we have

$$
\begin{gathered}
\sum_{x>0} \int_{-\pi}^{\pi} \frac{d p}{2 \pi}\left(\psi_{\kappa}(x) e^{-i p((1+w / 2) x+w / 4)}\right. \\
\left.-\eta^{R}(\kappa) \psi_{\kappa}(x) e^{-i p x}\right) \sigma_{p}=0
\end{gathered}
$$

where we are denoting

$$
\sigma_{p \geq 0}=a_{p}, \quad \sigma_{p<0}=-b_{p}^{\dagger}
$$

to avoid clutter.

Therefore the eigen-wave-function must take the form

$\sum_{x>0} \int_{-\pi}^{\pi} \frac{d p}{2 \pi}\left(\psi_{\kappa}(x) e^{-i p((1+w / 2) x+w / 4)}-\eta^{R}(\kappa) \psi_{\kappa}(x) e^{-i p x}\right)=0$.

Strictly speaking, the sum over $x$ leads to a delta function in the momenta which is only defined up to $2 \pi$. As we already noted, the boost operator only preserves the modes with a long wavelength compared to the lattice spacing $a$. In the limit $a \rightarrow 0$, the limits of the $p$ integral would be replaced by $\pm \pi / a \rightarrow \pm \infty$. Solutions to the equation are then given by the following:

$\tilde{\psi}_{\kappa}^{ \pm}(p)=\Theta( \pm p) p^{\kappa} e^{i \frac{p}{2}}, \quad \tilde{\psi}_{\kappa}^{ \pm}(p) \equiv \sum_{x} \psi_{\kappa}^{ \pm}(x) e^{-i p x}$.

Both lead to the same eigenvalue

$$
\eta^{R}(\kappa)=(1+w / 2)^{\kappa} .
$$

Note that the $e^{i p / 2}$ is from our fractional labels of sites $x=\frac{1}{2}, \frac{3}{2}, \cdots$. (In the $a \rightarrow 0$ limit this phase is really $e^{i p a / 2} \rightarrow 1$. We can neglect this factor when we consider the long-wavelength mode.) This is a solution in the rhs of the lattice where $x>0$. We note that $p$ can be both positive and negative here. Therefore, to recover the wave function in configuration space, we can in fact take an inverse transform, which gives

$$
\psi_{\kappa}^{ \pm}(x>0)=\int_{-\infty}^{\infty} \frac{d q}{2 \pi} e^{i q x} \tilde{\psi}_{\kappa}^{ \pm}(q) .
$$

Now, one can check that

$\psi_{\kappa}^{+}(x>0)=\int_{0}^{\infty} \frac{d p}{2 \pi} p^{\kappa} e^{i p x}=(-i x)^{-\kappa-1} \frac{\Gamma(1+\kappa)}{2 \pi}$. 
Here, it is necessary that $-1<\operatorname{Re} \kappa<0$ while assuming $x$ is real. This computation is not regulated based on giving a small imaginary part to $x$ which would not have been a good regulator if $p<0$. We would also like to check the result for $\psi_{\kappa}^{-}(x>0)$. It gives

$\psi_{\kappa}^{-}(x>0)=\int_{-\infty}^{0} \frac{d p}{2 \pi} p^{\kappa} e^{i p x}=(-1)^{\kappa}(i x)^{-\kappa-1} \frac{\Gamma(1+\kappa)}{2 \pi}$.

This means that $\psi_{\kappa}^{-}(x>0)$ is proportional to $\psi_{\kappa}^{+}(x>0)$ and does not lead to a new eigenfunction.

We can work out the eigenmodes in $x<0$ in a similar manner. As we mentioned above, the $x<0$ sector is the mirror image of the $x>0$ sector. (See Fig. 6.) This immediately suggests that we have a set of eigenmodes given by

$$
A_{\xi}^{L}=\sum_{x<0} \chi_{\xi}(x) a_{2 x}
$$

where $\chi_{\xi}(x)$ satisfies an entirely analogous set of eigenvalue equations (51).

We would then end up with the solutions

$$
\tilde{\chi}_{\xi}(p) \equiv \sum_{x<0} e^{-i p x} \chi_{\xi}(x),
$$

where

$$
\tilde{\chi}_{\xi}^{ \pm}(p)=p^{\xi} e^{-i \frac{p}{2}} \Theta( \pm p), \quad \eta^{L}(\xi)=(1+w / 2)^{\xi} .
$$

Now this recovers

$$
\chi_{\xi}(x<0)=\int_{-\infty}^{\infty} \frac{d q}{2 \pi} e^{i q x} \tilde{\chi}_{\xi}(q) .
$$

An important question then arises. What values should $\kappa$ and $\xi$ take? Recall that we are taking these infinite ladders as a unitary evolution. Therefore, $\kappa$ and $\xi$ should be chosen such that we have a pure phase.

We therefore would like to have

$$
\eta^{R}(\kappa)=(1+w / 2)^{\kappa}=e^{\eta \kappa}=e^{-i \eta \epsilon} .
$$

In other words,

$$
\kappa=-i \epsilon,
$$

for positive $\epsilon$, would define positive energy modes.

Comparing (58) with its counterpart in continuous quantum field theory we can set

$$
\xi=i \epsilon,
$$

for positive $\epsilon$ to correspond to positive energy modes. We interpret here that the unit of time evolution is evolved backward, and so $\Delta t$ has to take a negative value, thus inverting the definition of positive energies. Here, we labor further upon some ambiguity that creeps in. There is not an exact translation symmetry with respect to the evolution between ladders of different slopes-the analogue of evolution of Rindler observers here. As a result, there is not a precise concept of conserved energy (within the "energy Brillouin zone") here. Nonetheless, there is an approximate symmetry, so that we can still define $0 \leq \epsilon \ll 1$ to be approximately conserved positive energy modes in this analogue "Rindler" frame. Correspondingly $-1 \ll \epsilon \leq 0$ defines negative energy modes. That this "Rindler Hamiltonian" actually does approximate the entanglement Hamiltonian and thus bears actual resemblance to the continuous scenario with exact Killing vectors is discussed in numerical computations and subsequently in the discussions of integrable models in the accompanying paper [14].

In summary, we have four sets of wave functions, two independent ones for the $x>0$ region and another two for the $x<0$ regions,

$$
\left\{\psi_{\kappa=-i \epsilon}^{+}(x>0), \psi_{\kappa=i \epsilon}^{+}(x>0)\right\} \quad\left\{\chi_{\xi=i \epsilon}^{+}(x<0), \chi_{\xi=-i \epsilon}^{+}(x<0)\right\},
$$

where they correspond to pairs of positive and negative wave functions in each region for each $\epsilon$. We also note that $\left(\psi_{\kappa=i \epsilon}^{+}\right)^{*}(x>0)$ is proportional to $\psi_{\kappa=-i \epsilon}^{+}(x>0)$ and that $\left(\chi_{\xi=-i \epsilon}^{+}\right)^{*}(x<0)$ is proportional to $\chi_{\xi=i \epsilon}^{+}(x<0)$. Because in the same region, the wave function of the negative energy mode should be the complex conjugate of the wave function of the positive energy mode, we use $\psi_{\kappa=-i \epsilon}^{+}(x>0), \chi_{\xi=i \epsilon}^{+}(x<0)$, and their complex conjugate to define four sets of eigenoperators:

$$
\begin{array}{ll}
A^{R}(\epsilon)=\sum_{x>0} \psi_{-i \epsilon}^{+}(x) a_{2 x}, & B^{R^{\dagger}}(\epsilon)=\sum_{x>0}\left(\psi_{-i \epsilon}^{+}\right)^{*}(x) a_{2 x}, \\
A^{L}(\epsilon)=\sum_{x<0} \chi_{+i \epsilon}^{+}(x) a_{2 x}, & B^{L \dagger}(\epsilon)=\sum_{x<0}\left(\chi_{+i \epsilon}^{+}\right)^{*}(x) a_{2 x},
\end{array}
$$

where we have assumed that $\epsilon$ is positive. The complex conjugates of each operator can be obtained from the above. Entirely analogous expressions, albeit looking less clean and simple, can be obtained for modes on the even links, which we will not dwell on in detail here.

Before we move on to a detailed discussion of the Unruh effect in the current context, we note that the boost operator constructed from an ever rising sequence of stairs is crucial to the decoupling between the left and right moving modes which can be thought of as arising from a "Rindler horizon" near the origin. Initially we attempted to approximate this effect with a stair with only one step. This is equivalent to solving a semi-infinite system with a fixed boundary condition at one end. One can show that there is always reflection at $x=0$, leading to very different physics. 


\section{Approximating the Unruh effect}

Having worked out the eigenfunctions, we can follow a very similar path as the continuous case and look for the Bogoliubov transformation that connects the eigenmodes of the "inertial observers" and the "Rindler observers."

Equation (51) is actually supplying the Bogoliubov transformations between modes in the inertial frames and Rindler frames.

We would like to express $a_{p}$ in terms of $A^{R}(\epsilon)$ and $B^{L^{\dagger}}(\epsilon)$. We note that

$\chi_{i \epsilon}^{+}(x<0)=\int_{-\infty}^{\infty} \frac{d p}{2 \pi} p^{-i \epsilon} e^{-i p x} \Theta(p)=(-1)^{1-i \epsilon} \psi_{-i \epsilon}^{+}(x<0) ;$

i.e., in the last equality, the mode corresponds to $\psi_{i \epsilon}^{+}$, but analytically extended in the region $x<0$. This gives

$$
\begin{aligned}
A^{R}(\epsilon)+(-1)^{i \epsilon-1}\left(A^{L}(\epsilon)\right)^{\dagger} & =\int_{-\infty}^{\infty} \frac{d p}{2 \pi} \sum_{x} \Theta(p) p^{-i \epsilon} e^{i p x} a_{2 x} \\
& =\int_{0}^{\infty} \frac{d p}{2 \pi} p^{-i \epsilon} a_{p} .
\end{aligned}
$$

Here, one has to make a choice for the branch cut. If we make a choice that is

$$
(-1)^{i \epsilon}=\exp (i \pi *(i) \epsilon)=\exp (-\pi \epsilon),
$$

we would recover the standard result that the temperature for the half-space observer is given by ${ }^{3}$

$$
\hat{T}=\frac{1}{2 \pi}
$$

This can be compared with the standard result for relativistic field theories (in $1+1$ dimensions) at vanishing mass. A detailed computation can be found, for example, in [16], where we have

$$
\begin{aligned}
\alpha_{l>0}^{R} & =\int_{0}^{\infty} \frac{d k}{2 \pi} \frac{l}{k}\left(a_{k} f_{l}(k)+a_{k}^{\dagger} f_{l}(-k)\right), \\
f_{l}(k) & =k^{i l} a^{-i l-1} e^{\pi l / 2} \Gamma[-i l],
\end{aligned}
$$

where $\alpha_{l}^{R}$ are (positive energy bosonic) Rindler modes and $a_{k}$ are inertial modes. The speed of light and acceleration has been set to 1 . The solution for $f_{l}(k)$ is, up to normalization, precisely what we have found in (51). The fact that the modes are bosonic only changes the analysis concerning normalizations. Otherwise the relationships between the modes are a direct result of Fourier transforms, and the plane wave solutions are shared between the $1+1$-dimensional fermions and bosons.

\footnotetext{
${ }^{3}$ We note that the temperature is measured against our units of time, which have been set to 1 .
}

What makes our analysis interesting is that the same physics is recovered purely based on the discrete recursion relations following from the discrete boost operator. We have made no reference to Killing vectors or solving the Dirac equation in Rindler coordinates.

The even links can be treated in a similar manner, although the result would not have looked as clean due to our breaking of symmetries between even and odd links.

\section{Comparison with half-space entanglement}

What we have demonstrated in the previous subsection is that we can construct an analogue of a boost operator. Its effect on the modes in the inertial frame is very much analogous to the expected form in the continuous case, $a_{p} \rightarrow a_{\Lambda^{-1}}$, and this action (approximately) preserves the notion of positive and negative modes that we have defined in the discrete spacetime.

Then we showed that eigenmodes of this boost operator have very similar forms to the Rindler modes in continuous field theories. We demonstrated this explicitly at special values of the parameters and showed that they closely approximate modes solved in Rindler space. Subsequently we defined an analogous Bogoliubov transformation between inertial and Rindler modes.

What we have not demonstrated, however, is that the boost operator that we have defined is indeed the same as the entanglement Hamiltonian controlling half-space entanglement. In the case of a continuous field theory with Lorentz invariance, this is guaranteed by the Bisognano-Wichmann theorem [17] (see a review, for example, in [18]). The construction relies heavily on the analytic continuation of the boost operator that rotates $t \rightarrow-t$ and $x \rightarrow-x$.

We note that inspecting more generic integrable models, such an analytic continued operator indeed exists and can be readily constructed-it is the Euclidean version of the "corner transfer matrix" [14].

In this section however, we will compute explicitly the half-space entanglement Hamiltonian in the fermionic model for different values of $c$ and demonstrate that the modular Hamiltonian does resemble the guess of a boost operator that we made in the previous subsection, therefore adding extra evidence that the boost operator that we have constructed does indeed control the half-space entanglement. In that case, the evolution generated by our boost does correspond to the evolution of observers that approximately have no access to half of the spacetime, closely resembling Rindler observers.

\section{The computation of the entanglement Hamiltonian}

The entanglement Hamiltonian, which is also called the modular Hamiltonian, is defined by taking the log of the reduced density matrix. In the case of free fermions, given that the vacuum state we have chosen has zero fermion number, the reduced density matrix takes the following form: 


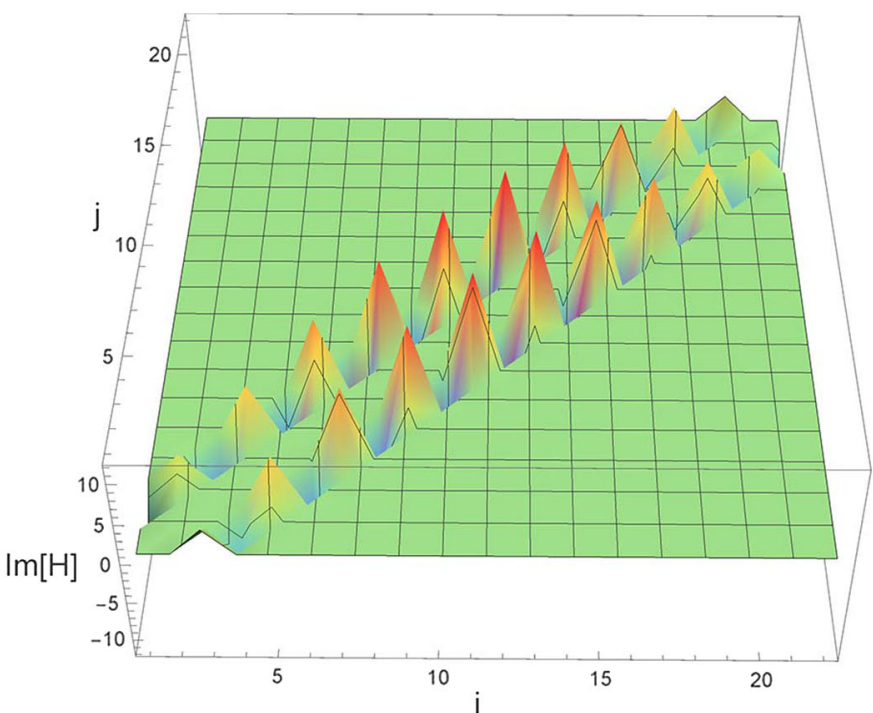

(a)

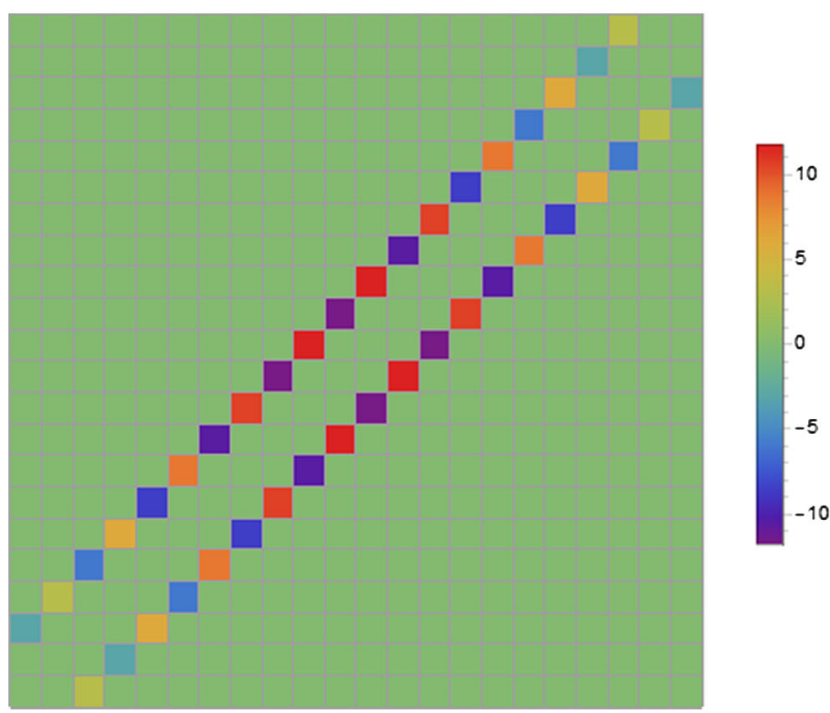

(b)

FIG. 7. Plots of the real part of the coefficients of $\left(a_{i}^{\dagger} a_{j}\right) / i$ terms in the Hamiltonian with $c=0.000001$ : The dominant contributions correspond to pairs of creation and annihilation operators located at positions $|i-j|=2$, where $i, j$ are the labels of links. (b) Vertical view of panel (a) that describes the values by color. The parameter $c$ is chosen to be 0.000001 . We note that neighboring peaks appear to take opposite signs. That is because even and odd sites decouple, and their respective peaks are out of phase.

$$
\rho_{V}=K e^{-\mathcal{H}}=K e^{-\sum_{V} \mathcal{H}_{i j} a_{i}^{\dagger} a_{j}}
$$

where $\rho_{V}$ is the reduced density matrix of region $V$ and $\mathcal{H}$ is the entanglement Hamiltonian of region $V$. The normalization constant is specified as $K=\operatorname{det}\left(1+e^{-\mathcal{H}}\right)^{-1}$. In [19], the entanglement Hamiltonian is calculated by the correlation functions of local creation and annihilation operators $a_{i}^{\dagger}, a_{j}$, which satisfy $\left\{a_{i}, a_{j}^{\dagger}\right\}=\delta_{i j}$. The correlation functions are defined as

$$
\begin{gathered}
\left\langle 0\left|a_{i} a_{j}^{\dagger}\right| 0\right\rangle=C_{i j}, \quad\left\langle 0\left|a_{j}^{\dagger} a_{i}\right| 0\right\rangle=\delta_{i j}-C_{i j}, \\
\left\langle 0\left|a_{i} a_{j}\right| 0\right\rangle=\left\langle 0\left|a_{i}^{\dagger} a_{j}^{\dagger}\right| 0\right\rangle=0 .
\end{gathered}
$$

The entanglement Hamiltonian in matrix form is given as [19]

$$
\mathcal{H}=-\log \left(C^{-1}-1\right)
$$

where $C$ is the matrix of correlation functions $C_{i j}$ in region $V$.

We demonstrate a plot of the modular Hamiltonian in Fig. 7 for $c \rightarrow 0$, which is the case considered in detail in the previous section. ${ }^{4}$

These plots suggest that the entanglement Hamiltonian takes the form

\footnotetext{
${ }^{4}$ In our analysis, we take region $V$ as the links connected with sites labeled from $1 / 2$ to $L-1 / 2$, i.e., links from 1 to $2 L$.
}

$$
\begin{aligned}
\mathcal{H}= & \frac{\mathcal{N}}{i} \sum_{x}\left(\frac{x(L-x)}{2 L}+\nu_{0}\right)\left(a_{2 x}^{\dagger} a_{2 x+2}-a_{2 x+2}^{\dagger} a_{2 x}\right) \\
& +(\text { even sublinks }) \ldots, \quad \nu_{0} \sim \frac{1}{10}
\end{aligned}
$$

for $c \rightarrow 0$.

This operator in (74) has been discussed before in the literature for the entanglement Hamiltonian of ground state lattice fermions with continuous time. In the computation of entanglement entropy of lattice fermionic Gaussian states, it is observed that the entanglement Hamiltonian always commutes with an operator of the above form $[20,21]$. Here, we are observing that it is itself a fair approximation of the entanglement Hamiltonian, at least in the $c \rightarrow 0$ or $c \rightarrow 1$ limit. The intermediate values of $c$ have extra complications as the translation symmetry of the ground state changes.

We show the fitted curve on top of the plot of the modular Hamiltonian in Fig. 8, showing that (74) is a close approximation of the modular Hamiltonian.

When we are sufficiently close to the entanglement boundary, one can see that it is approximately taking the form

$$
\mathcal{H} \approx \frac{\sigma}{2 i} \sum_{n} n\left(a_{n}^{\dagger} a_{n+1}-a_{n+1}^{\dagger} a_{n}\right)
$$

for some constant $\sigma$ and for $n$ sufficiently small and close to the boundary of the entanglement cut. We reckon this closely resembles the construction of the finite boost 


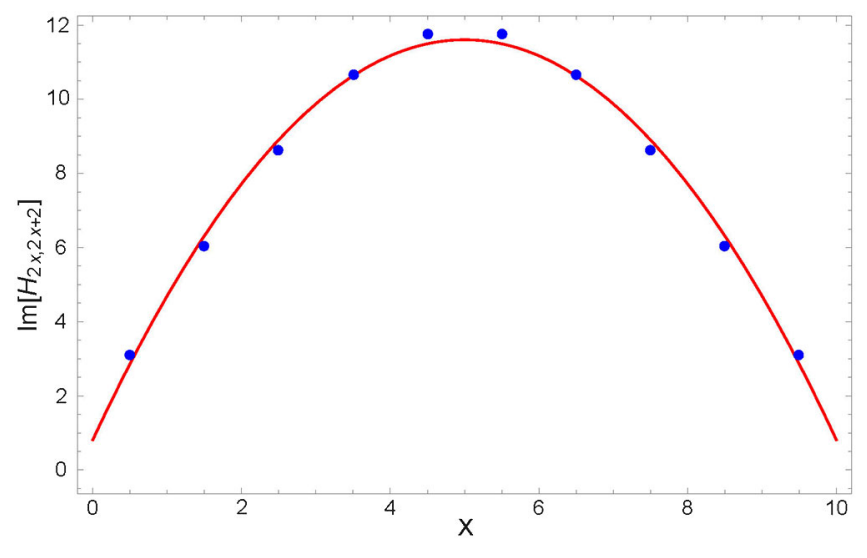

FIG. 8. Fitting of peaks of the imaginary part of the modular Hamiltonian with $c=0.000001$ and $L=11$. In this figure, we fit the peaks of the imaginary part of the modular Hamiltonian with a quadratic polynomial $a x^{2}+b x+f$, where $2 x$ is the label of a link. The fitting coefficients are $a=-0.431484, b=4.31484, f=0.817762$.

operator that we constructed, where the amount of time evolution grows linearly with the distance from the entanglement cut located at $x=0$. In a continuous Lorentz invariant theory, the vacuum entanglement Hamiltonian of the half-space is famously known to be given by the boost operator, which is given by $K=$ $\int d x x T_{00}$ at the $t=0$ slice. Therefore, the tensor network is naturally recovering this result, albeit only approximately. Our naive guess of the boost appears to match the actual entanglement Hamiltonian reasonably well, for different values of $c$, not restricted only to $c \rightarrow 0$, even though we solved the spectra of the boost operator only in that limit.

In the discussion below, we will inspect the commutation relations between the entanglement Hamiltonian and the creation/annihilation operators $a_{p}$, and we show that the algebra closely resembles our naive guess.

\section{Computing the algebra of the entanglement Hamiltonian}

The half-space entanglement Hamiltonian is only defined within the positive half line. We can complete the operator into one that acts on all of space by considering $\mathcal{H}-\overline{\mathcal{H}}$, where $\overline{\mathcal{H}}$ is the entanglement Hamiltonian of the complement of the region corresponding to $\mathcal{H}$, in this case, therefore, the "negative half region." We consider the commutator $\left[\mathcal{H}-\overline{\mathcal{H}}, a_{p}\right]$, i.e., links connected on sites $x=-L+\frac{1}{2}, \ldots,-\frac{1}{2}$.

To make a comparison with the boost operator that we constructed explicitly, we will particularly try to obtain the algebra of the entanglement Hamiltonian obtained above for $c \rightarrow 0$. Also, in the limit that $L$ is very large, we assume that we can ignore the corrections to the entanglement
Hamiltonian that arise from the other entangling boundary in a finite system. ${ }^{5}$

In this limit, the entanglement Hamiltonian therefore takes the form

$$
\begin{aligned}
\Gamma & \equiv(\mathcal{H}-\overline{\mathcal{H}})_{c \rightarrow 0, L \rightarrow \infty} \\
& =\frac{\mathcal{N}}{2 i} \sum_{x=-\infty}^{\infty}(x-\nu)\left(a_{2 x}^{\dagger} a_{2(x+1)}-a_{2(x+1)}^{\dagger} a_{2 x}\right),
\end{aligned}
$$

for some appropriate normalization $\mathcal{N}$, and $\nu$ is some constant.

We thus have

$$
\left[\Gamma, a_{p}\right]=\mathcal{N} \sum_{x=-\infty}^{\infty} a_{2 x} e^{i x p}\left(x \sin p+\frac{1}{2 i}\left((1-\nu) e^{-i p}+\nu e^{i p}\right)\right) .
$$

The $x \sin p$ term suggests that $\Gamma$ acts as $p \partial_{p} a_{p}$ in the leading long-wavelength limit, which is, up to a constant shift $\nu$, the infinitesimal version of the boost transformation that we introduced in (45).

\section{D. (Anti)commutation relations and correlation functions: Visualizing the light cone}

As a further check to the emergence of Lorentz invariance, particularly in the $c \rightarrow 0$ limit in which the Unruh effect can be solved analytically, we would like to inspect both the (anti)commutation relations and two point correlation functions evaluated with respect to the ground state that we have defined here. We would like to inspect the causal structures intrinsic to these tensor networks and their manifestation in the (anti)commutation relations and correlation function. It is also instructive to see how the boost symmetry manifests itself in the correlation functions, at least in some limits such as $c \rightarrow 0$ and $c \rightarrow 1$.

The anticommutation relations and correlation functions can be readily obtained. We relegate some of the details to the Appendix, while keeping only the main results. The complete expressions are presented in the Appendix.

(Anti)commutation relations are the most effective measure of causal structure, which is part of the Einstein locality axiom as discussed in Sec. III C 4. In Fig. 9, we can see that the anticommutators are exactly vanishing outside of the (effective) light cone for two choices of parameters corresponding to $c \rightarrow 0$, which displays emergent Lorentz

\footnotetext{
${ }^{5}$ There is subtlety with boundary conditions when dealing with the infinite system limit. In obtaining the reduced density matrix of any segment in a finite system, no matter how large the subsystem is taken to be within an even larger lattice, the pattern of the entanglement Hamiltonian is always sensitive to both boundaries. Our discussion here therefore has to be taken as a sanity check that the emerging pattern does have a set of recognizable physics.
} 


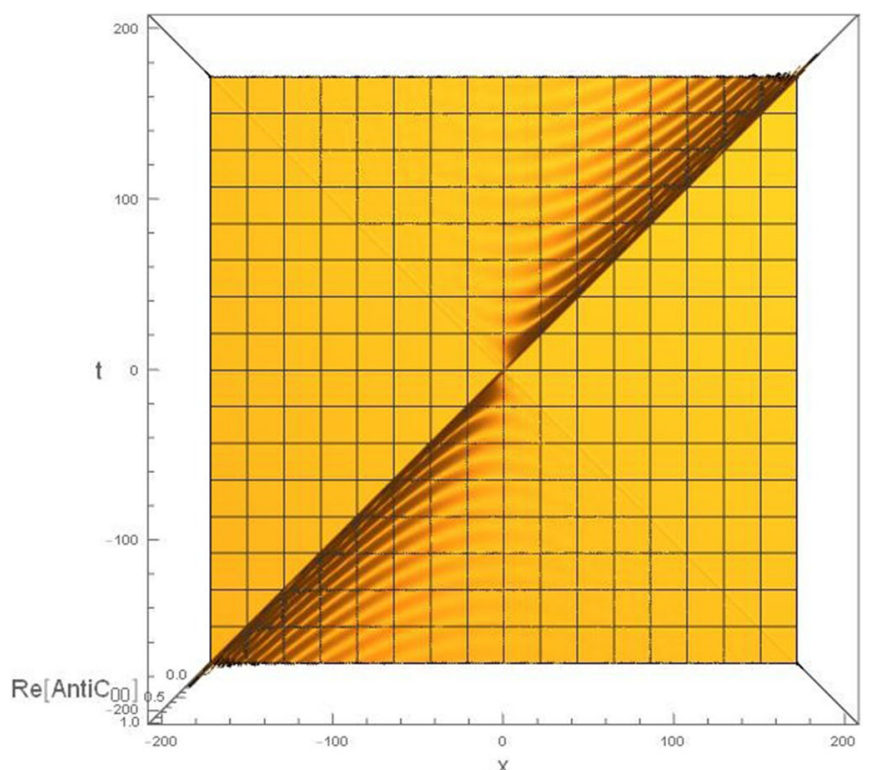

(a)

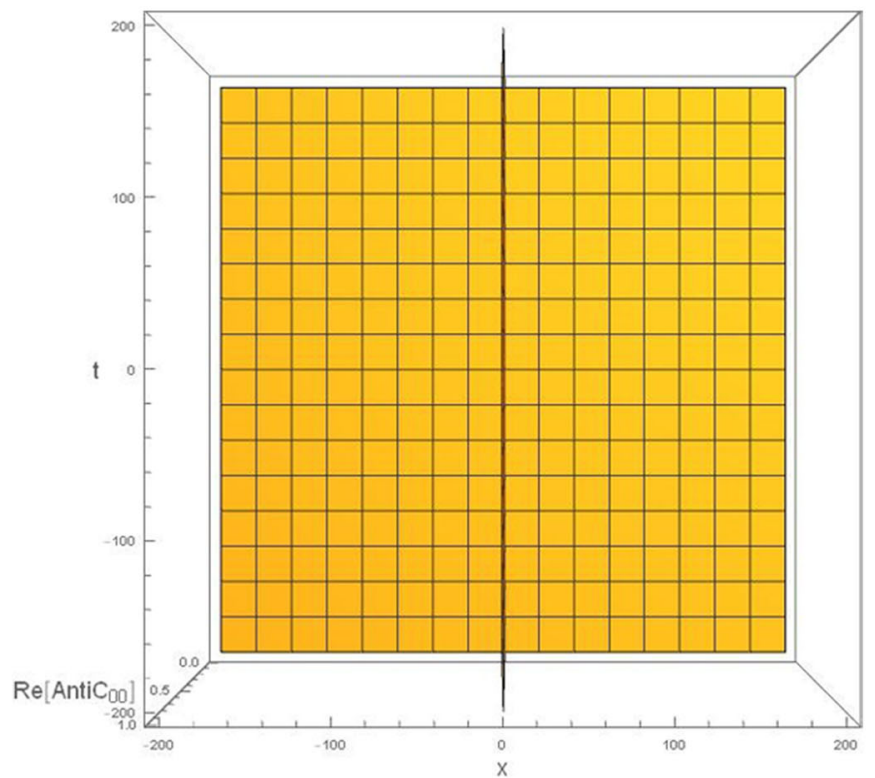

(c)

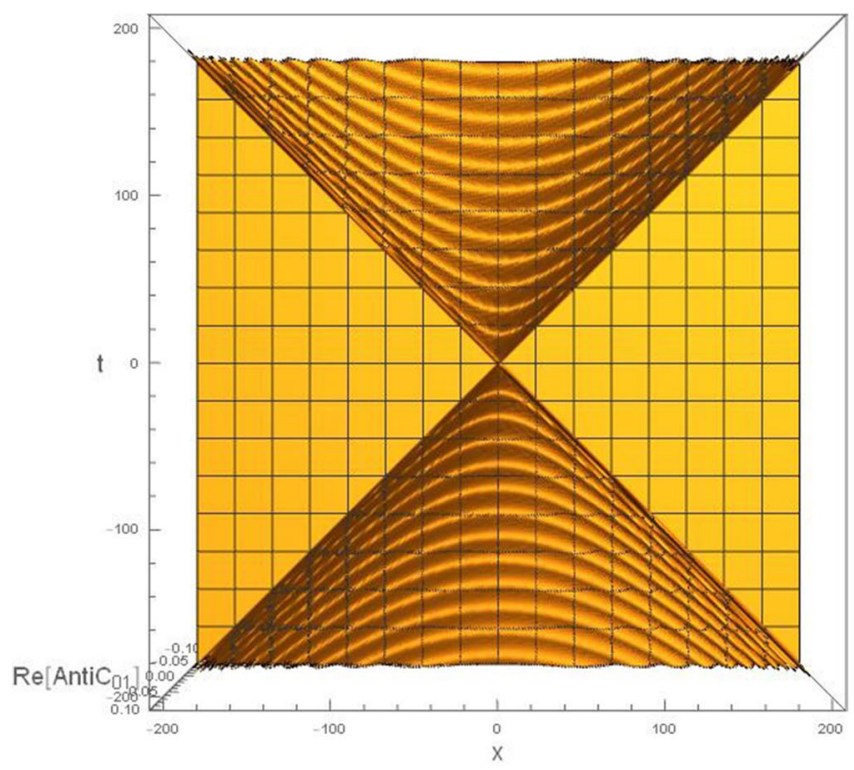

(b)

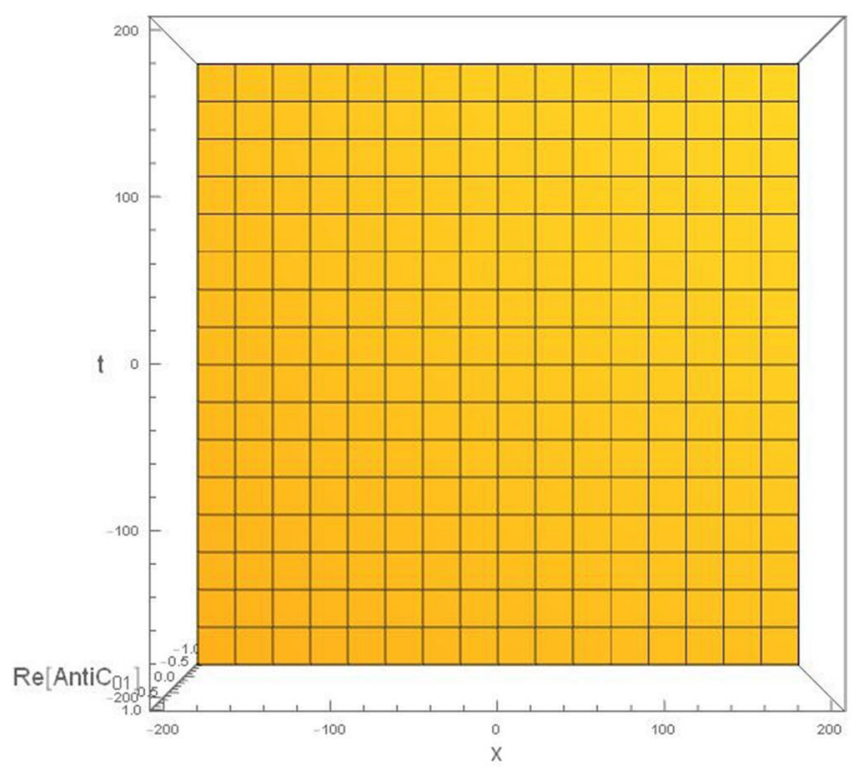

(d)

FIG. 9. Anticommutators of fermions. (a) Anticommutators $\left\{a_{2 x}(x, 0), a_{2 y}^{\dagger}(0, t)\right\}$ at $c=0.1$. (b) Anticommutators $\left\{a_{2 x}(x, 0), a_{2 y+1}^{\dagger}(0, t)\right\}$ at $c=0.1$. (c) Anticommutators $\left\{a_{2 x}(x, 0), a_{2 y}^{\dagger}(0, t)\right\}$ at $c=1$. (d) Anticommutators $\left\{a_{2 x}(x, 0), a_{2 y+1}^{\dagger}(0, t)\right\}$ at $c=1$, which is 0 in the whole spacetime.

invariance, and $c \rightarrow 1$, where the model becomes nondispersive. At $c=0$, since the dispersion relation is linear, the invariance under boost translates into an invariance of the anticommutators under a simple transformation in $(x, t)$ coordinates: namely, the Lorentz transformation. The hyperbolas marking "equipotential" lines are clearly visible in Fig. 9. There can be more symmetries in the dynamics than the graph would suggest for special choices of these evolution tensors. As $c \rightarrow 1$, the evolution is nondispersive with the light cone closing up. While the topology of the graph determines the maximal size of the light cone, their effective size depends on the actual evolution dictated by the choice of tensors populating the tensor network graph.

We have also looked into correlation functions which are contained in the Appendix B. There are various special limits in which the results are particularly illuminating. Again we focus on the $c \rightarrow 0$ limit, where each tensor behaves like a swap between the neighboring fermionic sites. We reproduce here the limit where $c \rightarrow 0$ : 


$$
\begin{gathered}
\left\langle 0\left|a_{2 x}(x, 0) a_{2 y}^{\dagger}(y, t)\right| 0\right\rangle=\mathcal{N}^{2} \sum_{p \geq 0} e^{-i p(x-y)} e^{i|p| t}, \\
\left\langle 0\left|a_{2 x+1}(x, 0) a_{2 y+1}^{\dagger}(y, t)\right| 0\right\rangle=\mathcal{N}^{2} \sum_{p<0} e^{-i p(x-y)} e^{i|p| t} .
\end{gathered}
$$

It is noteworthy that in (78), only half of the modes contribute. It is a direct consequence that in this limit, half of the links are decoupled from each other. Half of the links contribute to modes moving right, i.e., have positive energy for $p>0$; the other half contribute to left moving modes,

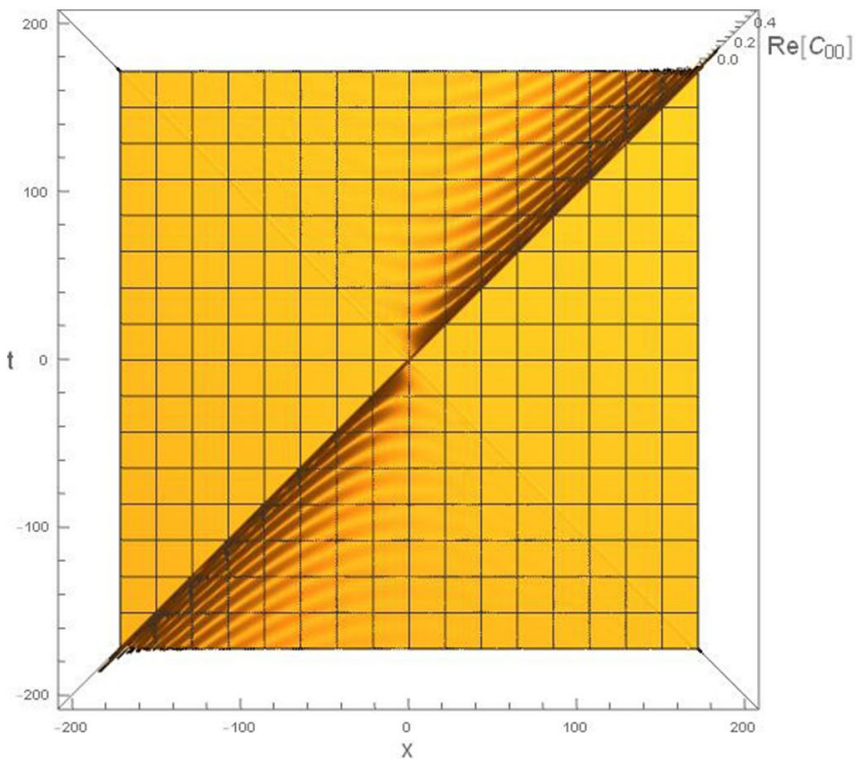

(a)

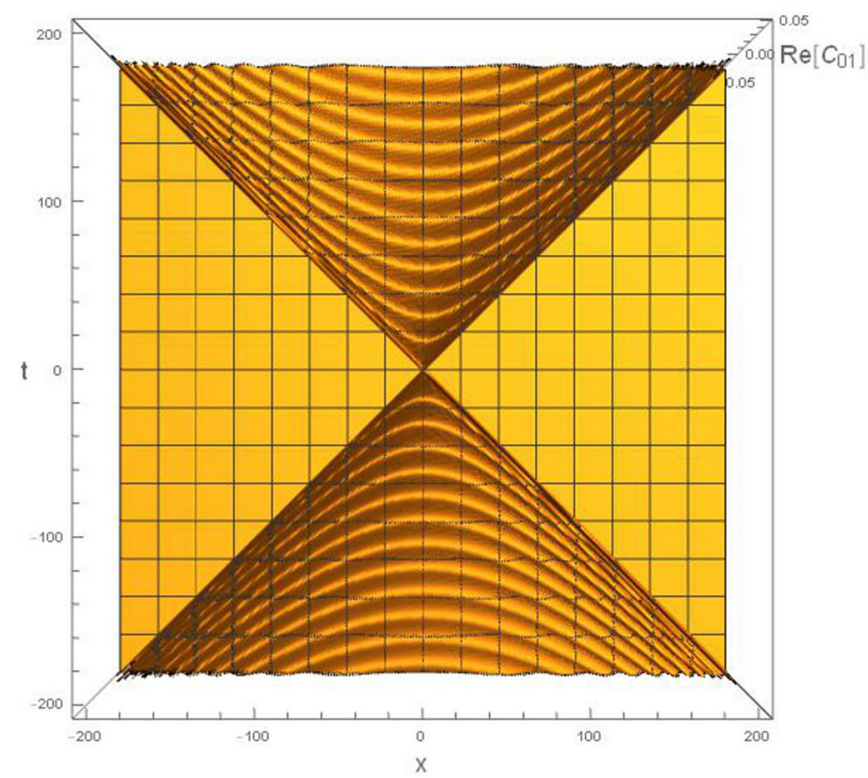

(c) with $p<0$ having positive energies. At the other end, where $c \rightarrow 1$, the time dependence completely drops out. The ground state, however, is chosen to have nontrivial entanglement, since it is still annihilated by half of the momentum eigenmodes taken as annihilation operators, as their eigenenergies tend to $0^{-}$in the nondispersive limit.

We note also that when we further take the limit $L \rightarrow \infty$ in the $c \rightarrow 0$ regime, the correlation functions reduce to the result of a free massless fermion in $1+1 \mathrm{~d}$.

In Figs. 10-12 we present the correlation functions corresponding to $c=0.1, c=s=1 / \sqrt{2}$, and $c=1$,

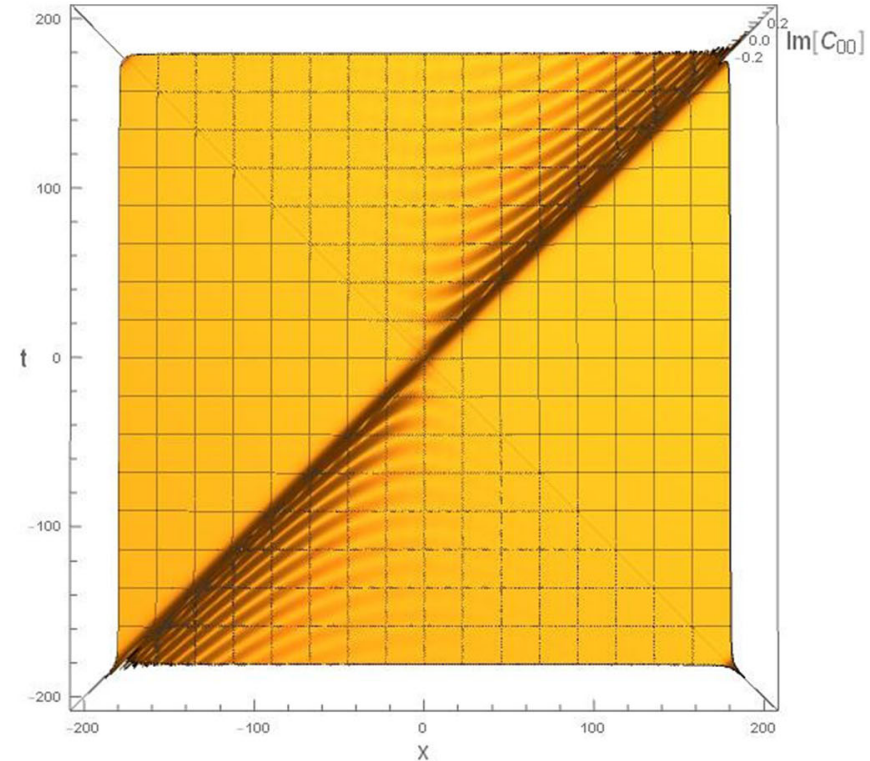

(b)

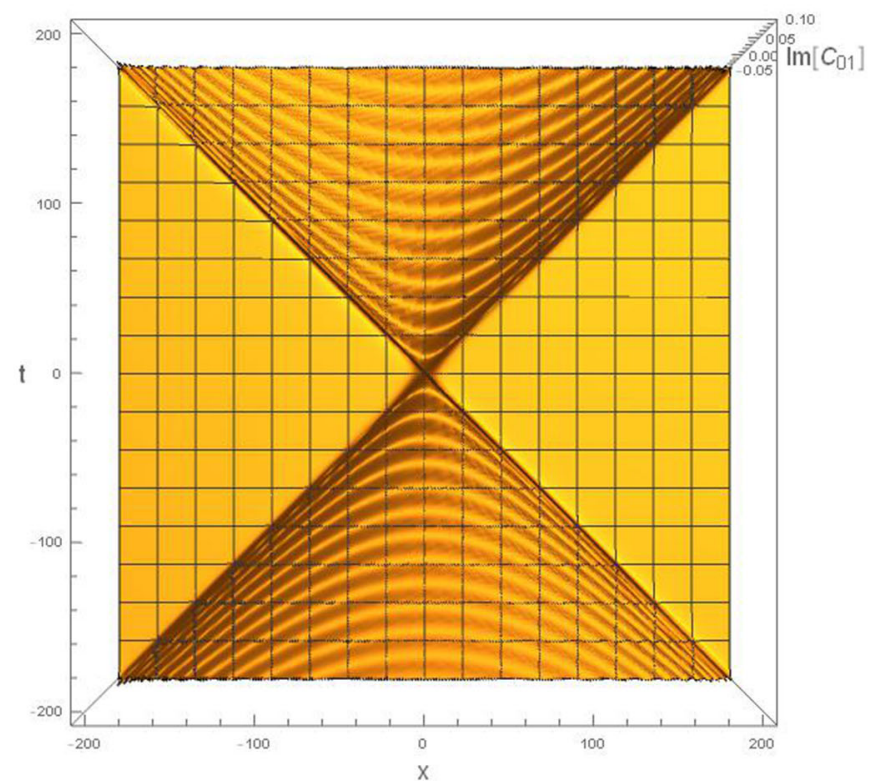

(d)

FIG. 10. Correlation functions evaluated at $c=0.1$. (a) Real part of correlation functions $\left\langle 0\left|a_{2 x}(x, 0) a_{2 y}^{\dagger}(0, t)\right| 0\right\rangle$. (b) Imaginary part of correlation functions $\left\langle 0\left|a_{2 x}(x, 0) a_{2 y}^{\dagger}(0, t)\right| 0\right\rangle$. (c) Real part of correlation functions $\left\langle 0\left|a_{2 x}(x, 0) a_{2 y+1}^{\dagger}(0, t)\right| 0\right\rangle$. (d) Imaginary part of correlation functions $\left\langle 0\left|a_{2 x}(x, 0) a_{2 y+1}^{\dagger}(0, t)\right| 0\right\rangle$. 


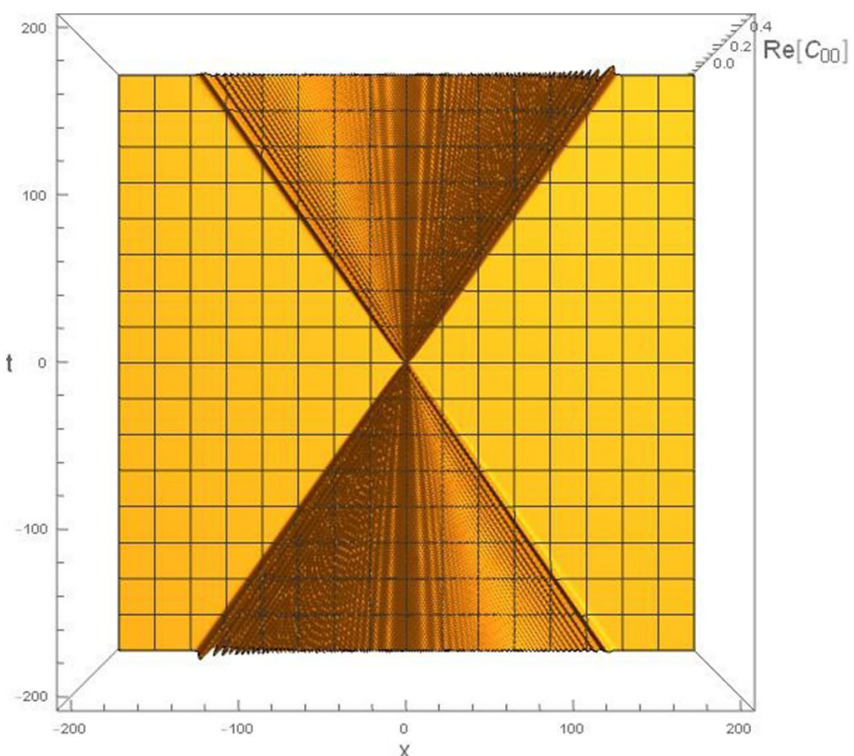

(a)

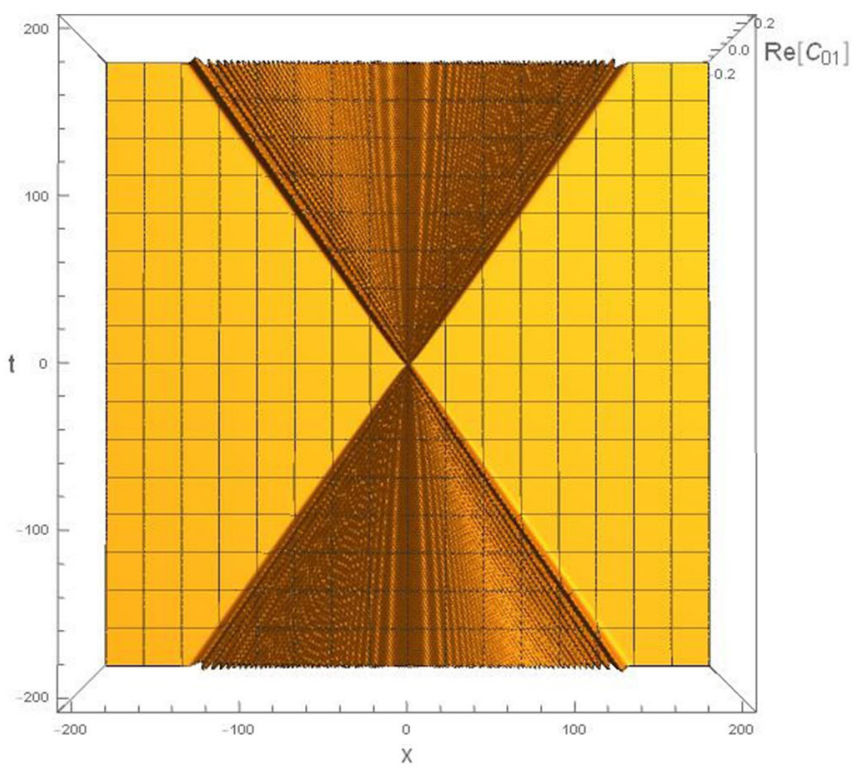

(c)

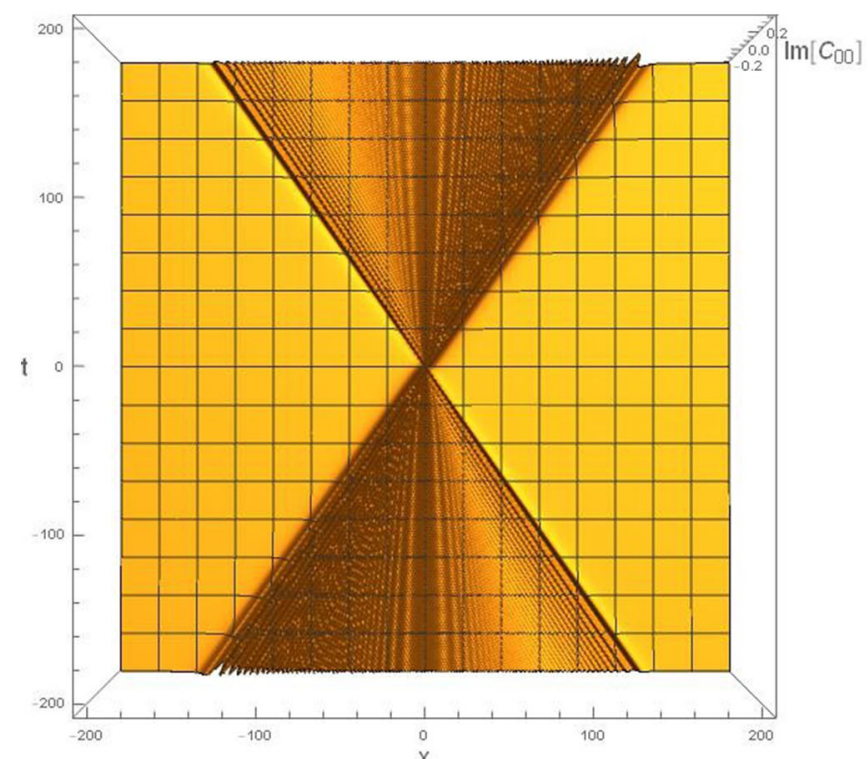

(b)

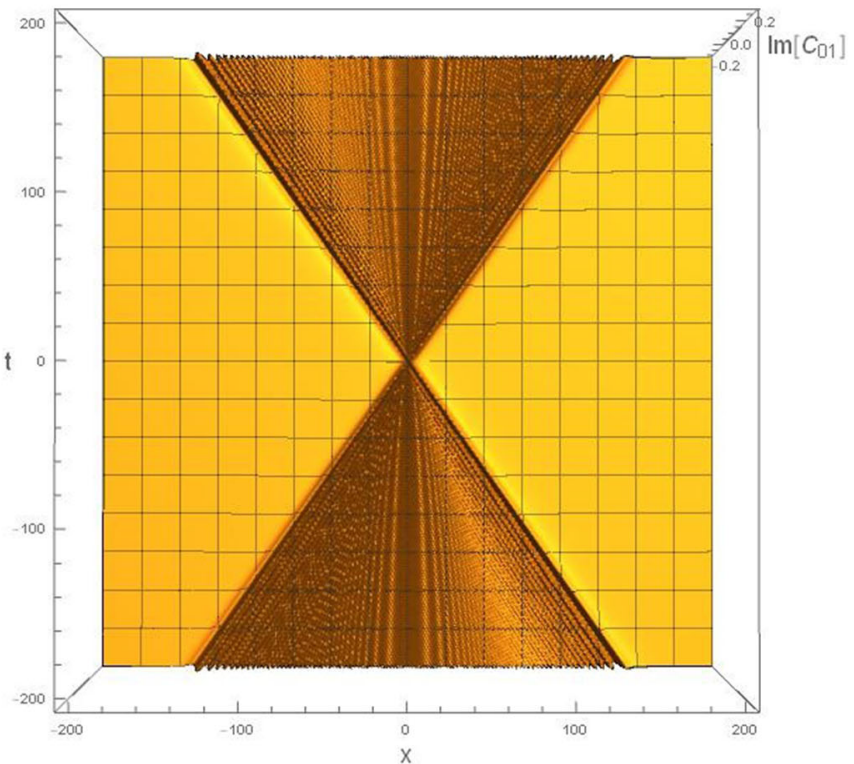

(d)

FIG. 11. Correlation functions evaluated at $c=s=1 / \sqrt{2}$. (a) Real part of correlation functions $\left\langle 0\left|a_{2 x}(x, 0) a_{2 y}^{\dagger}(0, t)\right| 0\right\rangle$. (b) Imaginary part of correlation functions $\left\langle 0\left|a_{2 x}(x, 0) a_{2 y}^{\dagger}(0, t)\right| 0\right\rangle$. (c) Real part of correlation functions $\left\langle 0\left|a_{2 x}(x, 0) a_{2 y+1}^{\dagger}(0, t)\right| 0\right\rangle$. (d) Imaginary part of correlation functions $\left\langle 0\left|a_{2 x}(x, 0) a_{2 y+1}^{\dagger}(0, t)\right| 0\right\rangle$.

respectively, for comparison. We use the notations $C_{00} \equiv$ $\left\langle 0\left|a_{2 x}(x, 0) a_{2 y}^{\dagger}(0, t)\right| 0\right\rangle$ and $C_{01} \equiv\left\langle 0\left|a_{2 x}(x, 0) a_{2 y+1}^{\dagger}(0, t)\right| 0\right\rangle$. The lattice is finite, where we take $L=200$. We can again see the light cone clearly in the figures, demonstrating the causal structure that follows immediately from the construction of the tensor network by local unitarity. Moreover, as already mentioned above, a given topology of the tensor network graph gives only the upper bound of the size of the light cone. The precise values that the tensor takes control the actual size of the light cone. At $c=1$ the light cone has shrunk to vanishing size as expected of a nondispersive theory. The regions outside of the light cones are generically not vanishing. They are decaying with a power law [see, for example, (B15)-(B17), (B27), and (B28)] and their structures are not so much visible when plotted next to the interior of the light cone where they take much greater values.

\section{E. Comments on bosons}

The sections above might have conveyed the message that it is always possible to build the network from a discretization of the continuous path integral, and recover 


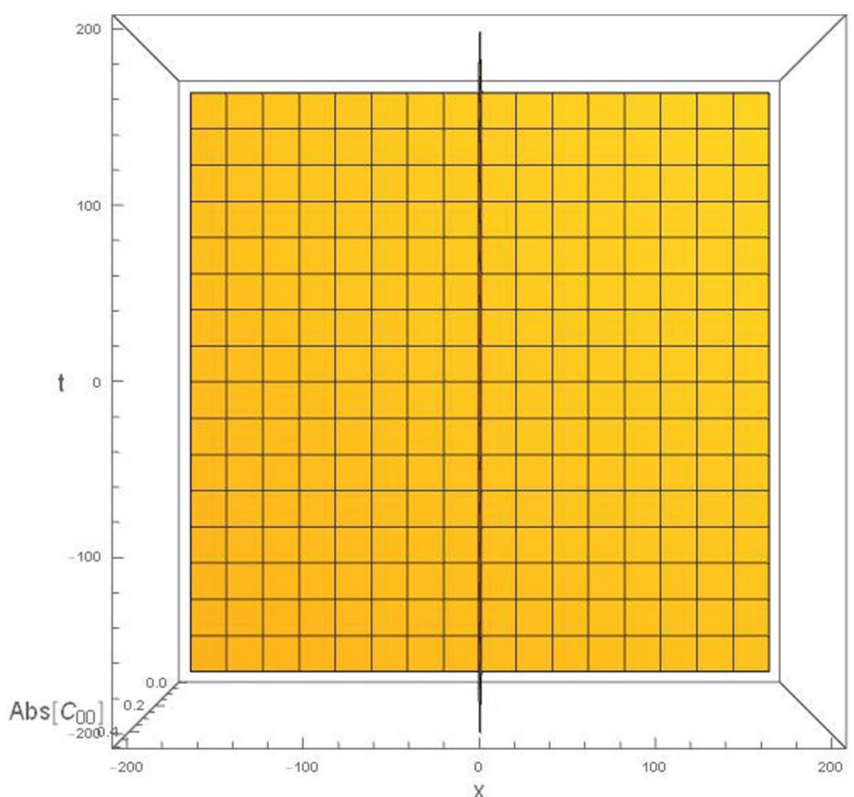

(a)

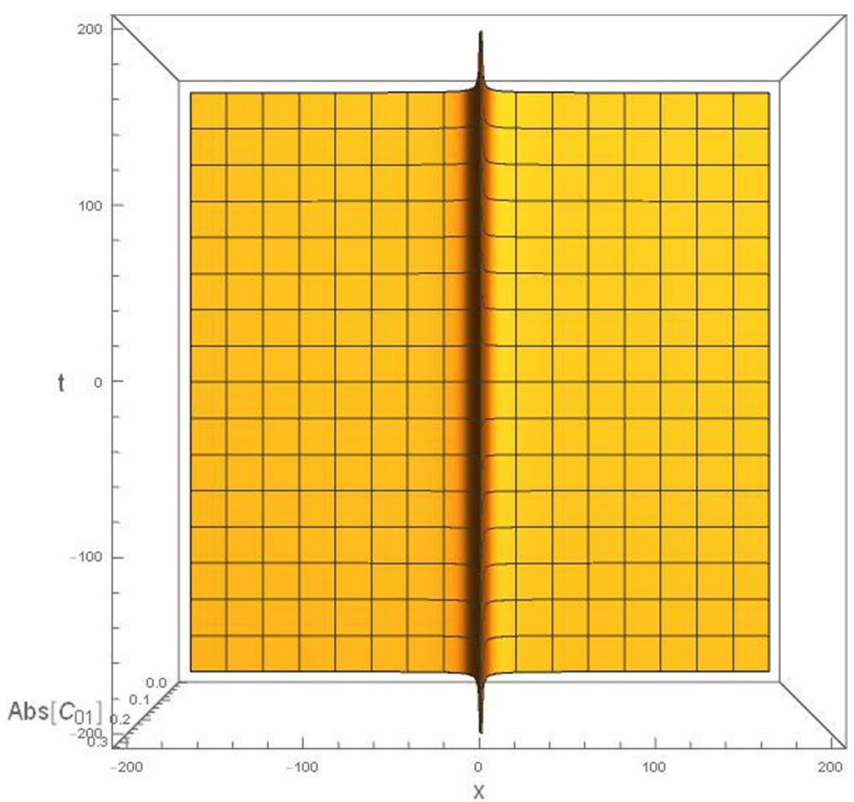

(b)

FIG. 12. Correlation functions evaluated at $c=1$. (a) Absolute value of correlation functions $\left\langle 0\left|a_{2 x}(x, 0) a_{2 y}^{\dagger}(0, t)\right| 0\right\rangle$. (b) Absolute value of correlation functions $\left\langle 0\left|a_{2 x}(x, 0) a_{2 y+1}^{\dagger}(0, t)\right| 0\right\rangle$.

the physics of the continuous field theory with some notion of a long-wavelength limit. There is, however, a reason why the paper focused on a discussion of Gaussian models of fermions rather than bosons. The authors have begun the journey with the hope of obtaining a tensor network of free bosons. Following almost exactly the same strategy as we have described for the fermions in this section, it is found that we invariably end up with a pair of eigenmodes, one satisfying the usual commutator $\left[a_{p}, a_{q}^{\dagger}\right]=\delta_{p, q}$, and the other pair satisfying the commutator with the wrong sign. This is somehow reminiscent of the issue encountered when quantizing bosons behind the black hole horizon. (See, for example, [22].) We have not understood the physical reasons for such a behavior other than the technical reasons why it ended up that way. It may be suggesting that there is potential obstruction to support particular kinds of operator algebra in a given network. This is not unheard of, as in the case of chiral fermions which are known to be impossible to simulate on a discrete (spatial) lattice. Discretizing both space and time in the case of a tensor network might suffer additional obstruction which is an interesting subject in its own right. We relegate the details of our (failed) attempt to model free bosons in a tensor network to the Appendix. Rather than lamenting an obstruction, the example emphasizes that the discretized model is intrinsically different from the continuous ones.

\section{CONCLUSIONS AND OUTLOOK}

In this paper, our goal is to explore the description of a Lorentzian spacetime via tensor networks. This is the first step towards building a controllable tensor network description of more exotic backgrounds, such as more generic curved backgrounds and perhaps ones mimicking AdS spaces and AdS black holes, which would shed light on a covariant understanding of the error correcting code/ tensor network description of the AdS/CFT correspondence which has achieved a great deal of success in static spacetimes [2,3].

We approached the problem by first comparing the framework of algebraic quantum field theory with the tensor network, and we found that the tensor network can very naturally fit into that picture, allowing one to formulate interesting questions about the tensor networks in very much the same language as is used in the AQFT. With inspirations from AQFT, we define notions of causality, Cauchy surfaces, and different frames, and we also specify the unitary transformations relating these observers.

In the second half of the paper, we considered explicit toy models based on fermions. In particular, as a first exercise, we illustrated that the physics of the Minkowski space can be captured to some extent in these simple settings, allowing one to explore questions such as the Unruh effect, which is a close relative of the Hawking temperature and Hawking radiation in black holes. In specific limits, the dispersion relations of our models show a clear signature of Lorentz invariance-the dispersion relation becomes linear without the doubling problem. We constructed a boost operator appropriate for the discrete spacetime and solved its spectra explicitly in the limit where dispersion approaches a linear one, and we demonstrated that they look completely parallel to modes observed by Rindler observers. We also gave support to this construction of the boost by comparing it with the 
actual half-space entanglement Hamiltonian which in the continuous case should indeed be equal to the boost operator.

Causal structure was subsequently studied based on anticommutation relations, invoking the Einstein locality axiom. One observes the emergence of a light cone that does not necessarily coincide with the same choice of graph. Not surprisingly, the effective light cone is controlled by the evolution tensors, which supply the "meat" of spacetime as the graph supplies a skeleton.

Our study serves as preparation for future explorations of more exotic features of quantum field theories in curved spacetimes. Further comprehensive studies in this subject have recently appeared, including [23,24] that supply complementary perspectives.

\section{ACKNOWLEDGMENTS}

We would like to thank Xiao-liang Qi for the suggestion to look at free fermion correlationfunctions instead of bosons. We would like to thank Hank Thacker, Yong-Shi Wu, and Guifre Vidal for very insightful discussions and suggestions. We would like to thank Muxin Han, Ce Shen, Gabriel Wong, and Jieqiang Wu for discussions and comments. We thank Chen-Te Ma for a critical and meticulous reading of our manuscript and a discussion on the doubling problem. We would also like to thank Jiawen Yan for processing figures. L. Y. H. and Z. Y. would like to thank the Tsinghua Sanya International Mathematics Forum for hospitality during the workshop and research-in-team program "Black holes, Quantum Chaos, and Solvable Quantum Systems," during which part of this work was undertaken. L. Y. H. acknowledges the support of Fudan University and the Thousand Young Talents Program. L. C. acknowledges support from China postdoctoral Science Foundation (Grant No. 2016M591593). A. B. would like to thank Professor Tadashi Takayanagi for useful discussions. A. B. is supported by a Japan Society for the Promotion of Science Grant-in Aid within a JSPS fellowship (Grant No. 17F17023).

\section{APPENDIX A: FREE BOSONS}

In this appendix, we will construct the tensor network that consists of free bosons.

As in the case of free fermions, we consider a set of bosonic creation and annihilation operators $a_{n}^{\dagger}$ and $a_{n}$ which satisfy the usual commutation relation

$$
\left[a_{n}, a_{n^{\prime}}^{\dagger}\right]=\delta_{n, n^{\prime}} .
$$

The Hamiltonian that generates the time evolution is

$H=\sum_{n=-2 L+1}^{2 L-1} h_{n-1, n}=\sum_{n=-2 L+1}^{2 L-1} \frac{1}{2 i}\left(a_{n-1}^{\dagger} a_{n}-a_{n}^{\dagger} a_{n-1}\right)$.
Again, over a sufficiently small unit of time $\Delta t$, the time evolution operator $U(\Delta t)$ can be well approximated as follows:

$$
U(\Delta t)=\left(\prod_{i} U_{2 i, 2 i+1}\right)\left(\prod_{j} U_{2 j-1,2 j}\right),
$$

where

$$
U_{i-1, i}=\exp \left(i \Delta t h_{i-1, i}\right) .
$$

Following the same procedure as in the fermion case, the eigenoperators are given by

$$
\begin{aligned}
& a_{p}=\sum_{n=-L+\frac{1}{2}}^{L-\frac{1}{2}}\left(\lambda_{1}^{+} \mathrm{e}^{i p n} a_{2 n-1}+\lambda_{2}^{+} \mathrm{e}^{i p n} a_{2 n}\right), \\
& b_{p}=\sum_{n=-L+\frac{1}{2}}^{L-\frac{1}{2}}\left(\lambda_{1}^{-} \mathrm{e}^{i p n} a_{2 n-1}+\lambda_{2}^{-} \mathrm{e}^{i p n} a_{2 n}\right),
\end{aligned}
$$

which follow from the eigenequations $U^{\dagger} a_{p} U=E a_{p}$ and $U^{\dagger} b_{p} U=E^{*} a_{p}$. Furthermore, the explicit forms for these eigenequations are

$$
\begin{gathered}
\frac{\lambda_{1}^{+}}{\lambda_{2}^{+}}=\frac{\mathrm{e}^{-i p} c s-c s}{c^{2}+s^{2} \mathrm{e}^{-i p}-E}=\frac{E-\mathrm{e}^{i p} s^{2}-c^{2}}{c s \mathrm{e}^{i p}-c s}, \\
\frac{\lambda_{1}^{-}}{\lambda_{2}^{-}}=\frac{\mathrm{e}^{-i p} c s-c s}{c^{2}+s^{2} \mathrm{e}^{-i p}-E^{*}}=\frac{E^{*}-\mathrm{e}^{i p} s^{2}-c^{2}}{c s \mathrm{e}^{i p}-c s},
\end{gathered}
$$

where $c$ and $s$ denote $\cos \Delta t / 2$ and $\sin \Delta t / 2$, respectively. It is straightforward to see that

$$
\frac{\lambda_{1}^{+}}{\lambda_{2}^{+}} \cdot \frac{\lambda_{1}^{-*}}{\lambda_{2}^{-*}}=-1 .
$$

From this one can immediately conclude that

$$
\left[a_{p}, b_{p^{\prime}}\right]=\left[a_{p}, b_{p^{\prime}}^{\dagger}\right]=0 .
$$

Beside this, the following relation has to hold,

$$
\lambda_{1}^{+} \lambda_{1}^{+*}+\lambda_{2}^{+} \lambda_{2}^{+*}=1,
$$

to get

$$
\left[a_{p}, a_{p^{\prime}}^{\dagger}\right]=\delta_{p, p^{\prime}} .
$$

But for the other pair we end up with

$$
\lambda_{1}^{-} \lambda_{1}^{-*}+\lambda_{2}^{-} \lambda_{2}^{-*}=1
$$


and

\section{APPENDIX B: (ANTI)COMMUTATORS AND CORRELATION FUNCTIONS OF THE \\ FERMIONIC TENSOR NETWORK MODEL}$$
\left[b_{p}^{\dagger}, b_{p^{\prime}}\right]=-\delta_{p, p^{\prime}} .
$$

We always end up with the opposite sign for this commutator, unlike for the fermionic case where both the signs are correct.
We relegate computations of the fermion tensor network to this appendix.

Below we list the results for the anticommutators of the fermions:

$$
\begin{gathered}
\left\{a_{2 x}(x, 0), a_{2 y}^{\dagger}(y, t)\right\}=\mathcal{N}^{2} \sum_{p} e^{-i p(x-y)}\left[e^{i|\chi| t}\left(\frac{c^{2}}{2\left(2 c^{2}+s^{2} \cos p+s^{2}\right)}+\frac{\left(s \sin p+\sqrt{(1-\cos p)\left(2 c^{2}+s^{2} \cos p+s^{2}\right)}\right)^{2}}{4(1-\cos p)\left(2 c^{2}+s^{2} \cos p+s^{2}\right)}\right)\right. \\
+e^{-i|\chi| t}\left(\frac{c^{2}}{2\left(2 c^{2}+s^{2} \cos p+s^{2}\right)}+\frac{\left(s \sin p-\sqrt{(1-\cos p)\left(2 c^{2}+s^{2} \cos p+s^{2}\right)}\right)^{2}}{4(1-\cos p)\left(2 c^{2}+s^{2} \cos p+s^{2}\right)}\right), \\
\left\{a_{2 x}(x, 0), a_{2 y+1}^{\dagger}(y, t)\right\}=-\mathcal{N}^{2} \sum_{p} e^{-i p(x-y)} 2 i \sin (|\chi| t) \frac{e^{i \frac{p}{2}} \sqrt{(1-\cos p)\left(2 c^{2}+s^{2} \cos p+s^{2}\right)}}{2 \sin \frac{p}{2}\left(2 c^{2}+s^{2} \cos p+s^{2}\right)}, \\
\left\{a_{2 x+1}(x, 0), a_{2 y}^{\dagger}(y, t)\right\}=-\mathcal{N}^{2} \sum_{p} e^{-i p(x-y)} 2 i \sin (|\chi| t) \frac{e^{-i \frac{p}{2}} c \sqrt{(1-\cos p)\left(2 c^{2}+s^{2} \cos p+s^{2}\right)}}{2 \sin \frac{p}{2}\left(2 c^{2}+s^{2} \cos p+s^{2}\right)}, \\
\left\{a_{2 x+1}(x, 0), a_{2 y+1}^{\dagger}(y, t)\right\}=\mathcal{N}^{2} \sum_{p} e^{-i p(x-y)}\left[e^{i|\chi| t}\left(\frac{c^{2}}{2\left(2 c^{2}+s^{2} \cos p+s^{2}\right)}+\frac{\left(s \sin p-\sqrt{(1-\cos p)\left(2 c^{2}+s^{2} \cos p+s^{2}\right)}\right)^{2}}{4(1-\cos p)\left(2 c^{2}+s^{2} \cos p+s^{2}\right)}\right)\right. \\
+e^{-i|\chi| t}\left(\frac{c^{2}}{2\left(2 c^{2}+s^{2} \cos p+s^{2}\right)}+\frac{\left(s \sin p+\sqrt{(1-\cos p)\left(2 c^{2}+s^{2} \cos p+s^{2}\right)}\right)^{2}}{4(1-\cos p)\left(2 c^{2}+s^{2} \cos p+s^{2}\right)}\right)
\end{gathered}
$$

Below we list results for the correlation functions of the fermions:

$$
\begin{gathered}
\left\langle 0\left|a_{2 x}(x, 0) a_{2 y}^{\dagger}(y, t)\right| 0\right\rangle=\mathcal{N}^{2} \sum_{p} e^{-i p(x-y)} e^{i|\chi| t}\left(\frac{c^{2}}{2\left(2 c^{2}+s^{2} \cos p+s^{2}\right)}+\frac{\left(s \sin p+\sqrt{(1-\cos p)\left(2 c^{2}+s^{2} \cos p+s^{2}\right)}\right)^{2}}{4(1-\cos p)\left(2 c^{2}+s^{2} \cos p+s^{2}\right)}\right) \\
\left\langle 0\left|a_{2 x}(x, 0) a_{2 y+1}^{\dagger}(y, t)\right| 0\right\rangle=-\mathcal{N}^{2} \sum_{p} e^{-i p(x-y)} e^{i|\chi| t} \frac{e^{i \frac{p}{2}} c \sqrt{(1-\cos p)\left(2 c^{2}+s^{2} \cos p+s^{2}\right)}}{2 \sin \frac{p}{2}\left(2 c^{2}+s^{2} \cos p+s^{2}\right)} \\
\left\langle 0\left|a_{2 x+1}(x, 0) a_{2 y}^{\dagger}(y, t)\right| 0\right\rangle=-\mathcal{N}^{2} \sum_{p} e^{-i p(x-y)} e^{i|\chi| t} \frac{e^{-i \frac{p}{2}} c \sqrt{(1-\cos p)\left(2 c^{2}+s^{2} \cos p+s^{2}\right)}}{2 \sin \frac{p}{2}\left(2 c^{2}+s^{2} \cos p+s^{2}\right)} \\
\left\langle 0\left|a_{2 x+1}(x, 0) a_{2 y+1}^{\dagger}(y, t)\right| 0\right\rangle=\mathcal{N}^{2} \sum_{p} e^{-i p(x-y)} e^{i|\chi| t}\left(\frac{c^{2}}{2\left(2 c^{2}+s^{2} \cos p+s^{2}\right)}+\frac{\left(s \sin p-\sqrt{(1-\cos p)\left(2 c^{2}+s^{2} \cos p+s^{2}\right)}\right)^{2}}{4(1-\cos p)\left(2 c^{2}+s^{2} \cos p+s^{2}\right)}\right)
\end{gathered}
$$

In the limit $c \rightarrow 0, s \rightarrow 1$, we have

$$
\left\langle 0\left|a_{2 x}(x, 0) a_{2 y}^{\dagger}(y, t)\right| 0\right\rangle=\mathcal{N}^{2} \sum_{p \geq 0} e^{-i p(x-y)} e^{i|p| t},
$$


$\left\langle 0\left|a_{2 x}(x, 0) a_{2 y+1}^{\dagger}(y, t)\right| 0\right\rangle=\left\langle 0\left|a_{2 x+1}(x, 0) a_{2 y}^{\dagger}(y, t)\right| 0\right\rangle=0$,

$\left\langle 0\left|a_{2 x+1}(x, 0) a_{2 y+1}^{\dagger}(y, t)\right| 0\right\rangle=\mathcal{N}^{2} \sum_{p<0} e^{-i p(x-y)} e^{i|p| t}$.

When $p$ is summed, the results become

$$
\begin{gathered}
\left\langle 0\left|a_{2 x}(x, 0) a_{2 y}^{\dagger}(y, t)\right| 0\right\rangle=\frac{1}{2 L} \frac{1-e^{-i \pi(x-y-t)}}{1-e^{-i \frac{\pi}{L}(x-y-t)}}, \quad \text { (B12) } \\
\left\langle 0\left|a_{2 x}(x, 0) a_{2 y+1}^{\dagger}(y, t)\right| 0\right\rangle=\left\langle 0\left|a_{2 x+1}(x, 0) a_{2 y}^{\dagger}(y, t)\right| 0\right\rangle=0,
\end{gathered}
$$

$\left\langle 0\left|a_{2 x+1}(x, 0) a_{2 y+1}^{\dagger}(y, t)\right| 0\right\rangle=\frac{1}{2 L} \frac{e^{i \pi(x-y+t)}-1}{1-e^{-i \frac{\pi}{L}(x-y+t)}}$.

When we take the limit $L \rightarrow \infty$, the above correlation functions become

$$
\left\langle 0\left|a_{2 x}(x, 0) a_{2 y}^{\dagger}(y, t)\right| 0\right\rangle=\frac{1-e^{-i \pi(x-y-t)}}{i 2 \pi(x-y-t)},
$$

$\left\langle 0\left|a_{2 x}(x, 0) a_{2 y+1}^{\dagger}(y, t)\right| 0\right\rangle=\left\langle 0\left|a_{2 x+1}(x, 0) a_{2 y}^{\dagger}(y, t)\right| 0\right\rangle=0$,

$$
\left\langle 0\left|a_{2 x+1}(x, 0) a_{2 y+1}^{\dagger}(y, t)\right| 0\right\rangle=\frac{e^{i \pi(x-y+t)}-1}{i 2 \pi(x-y+t)} .
$$

In the limit $c \rightarrow 1, s \rightarrow 0$, we have

$$
\begin{array}{r}
\left\langle 0\left|a_{2 x}(x, 0) a_{2 y}^{\dagger}(y, t)\right| 0\right\rangle=\frac{1}{2} \mathcal{N}^{2} \sum_{p} e^{-i p(x-y)}, \\
\left\langle 0\left|a_{2 x}(x, 0) a_{2 y+1}^{\dagger}(y, t)\right| 0\right\rangle \\
=-\mathcal{N}^{2} \sum_{p} e^{-i p(x-y)} \frac{e^{i \frac{p}{2}} \sqrt{2(1-\cos p)}}{4 \sin \frac{p}{2}}, \\
\left\langle 0\left|a_{2 x+1}(x, 0) a_{2 y}^{\dagger}(y, t)\right| 0\right\rangle \\
=-\mathcal{N}^{2} \sum_{p} e^{-i p(x-y)} \frac{e^{-i \frac{p}{2}} \sqrt{2(1-\cos p)}}{4 \sin \frac{p}{2}}, \\
\left\langle 0\left|a_{2 x+1}(x, 0) a_{2 y+1}^{\dagger}(y, t)\right| 0\right\rangle=\frac{1}{2} \mathcal{N}^{2} \sum_{p} e^{-i p(x-y)} .
\end{array}
$$

There is no time dependence in the correlation functions in the limit $c \rightarrow 1, s \rightarrow 0$, as expected of a theory with a trivial dispersion relation.

Summing $p$, the results become

$$
\left\langle 0\left|a_{2 x}(x, 0) a_{2 y}^{\dagger}(y, t)\right| 0\right\rangle=\frac{1}{2} \delta_{x y},
$$

$$
\begin{aligned}
\left\langle 0\left|a_{2 x}(x, 0) a_{2 y+1}^{\dagger}(y, t)\right| 0\right\rangle & =-\frac{1}{4 L} \frac{2-e^{-i \pi\left(x-y-\frac{1}{2}\right)}-e^{i \pi\left(x-y-\frac{1}{2}\right)}}{1-e^{-i \frac{\pi}{L}\left(x-y-\frac{1}{2}\right)}} \\
& =-\frac{1}{2 L} \frac{1}{1-e^{-i \frac{\pi}{L}\left(x-y-\frac{1}{2}\right)}}, \quad \text { (B23) }
\end{aligned}
$$

$$
\begin{aligned}
\left\langle 0\left|a_{2 x+1}(x, 0) a_{2 y}^{\dagger}(y, t)\right| 0\right\rangle & =-\frac{1}{4 L} \frac{2-e^{-i \pi\left(x-y+\frac{1}{2}\right)}-e^{i \pi\left(x-y+\frac{1}{2}\right)}}{1-e^{-i \frac{\pi}{L}\left(x-y+\frac{1}{2}\right)}} \\
& =-\frac{1}{2 L} \frac{1}{1-e^{-i \frac{\pi}{L}\left(x-y+\frac{1}{2}\right)}}, \quad \text { (B24) }
\end{aligned}
$$

$$
\left\langle 0\left|a_{2 x+1}(x, 0) a_{2 y+1}^{\dagger}(y, t)\right| 0\right\rangle=\frac{1}{2} \delta_{x y} .
$$

When we take the limit $L \rightarrow \infty$, the above correlation functions become

$$
\left\langle 0\left|a_{2 x}(x, 0) a_{2 y}^{\dagger}(y, t)\right| 0\right\rangle=\frac{1}{2} \delta(x-y),
$$

$$
\begin{aligned}
\left\langle 0\left|a_{2 x}(x, 0) a_{2 y+1}^{\dagger}(y, t)\right| 0\right\rangle & =-\frac{2-e^{-i \pi\left(x-y-\frac{1}{2}\right)}-e^{i \pi\left(x-y-\frac{1}{2}\right)}}{4 \pi i\left(x-y-\frac{1}{2}\right)} \\
& =-\frac{1}{2 \pi i\left(x-y-\frac{1}{2}\right)},
\end{aligned}
$$

$$
\begin{aligned}
\left\langle 0\left|a_{2 x+1}(x, 0) a_{2 y}^{\dagger}(y, t)\right| 0\right\rangle & =-\frac{2-e^{-i \pi\left(x-y+\frac{1}{2}\right)}-e^{i \pi\left(x-y+\frac{1}{2}\right)}}{4 \pi i\left(x-y+\frac{1}{2}\right)} \\
& =-\frac{1}{2 \pi i\left(x-y+\frac{1}{2}\right)},
\end{aligned}
$$

$$
\left\langle 0\left|a_{2 x+1}(x, 0) a_{2 y+1}^{\dagger}(y, t)\right| 0\right\rangle=\frac{1}{2} \delta(x-y) .
$$

As expected in a nondispersive system, there is no time dependence in any of the correlation functions. 
[1] B. Swingle, Entanglement renormalization and holography, Phys. Rev. D 86, 065007 (2012).

[2] F. Pastawski, B. Yoshida, D. Harlow, and J. Preskill, Holographic quantum error-correcting codes: Toy models for the bulk/boundary correspondence, J. High Energy Phys. 06 (2015) 149.

[3] P. Hayden, S. Nezami, X.-L. Qi, N. Thomas, M. Walter, and Z. Yang, Holographic duality from random tensor networks, J. High Energy Phys. 11 (2016) 009.

[4] X.-L. Qi and Z. Yang, Space-time random tensor networks and holographic duality, arXiv:1801.05289.

[5] C. Gattringer and C. B. Lang, Quantum chromodynamics on the lattice, Lect. Notes Phys. 788, 1 (2010).

[6] K. Fredenhagen and K. Rejzner, Quantum field theory on curved spacetimes: Axiomatic framework and examples, J. Math. Phys. (N.Y.) 57, 031101 (2016).

[7] R. M. Wald, Quantum Field Theory in Curved Space-Time and Black Hole Thermodynamics, Chicago Lectures in Physics (University of Chicago Press, Chicago, IL, 1995).

[8] L. Bombelli, J. Lee, D. Meyer, and R. Sorkin, Space-Time as a Causal Set, Phys. Rev. Lett. 59, 521 (1987).

[9] R. D. Sorkin, Causal sets: Discrete gravity, in Lectures on Quantum Gravity. Proceedings, School of Quantum Gravity, Valdivia, Chile, 2002 (2003), pp. 305-327.

[10] C. Beny, Causal structure of the entanglement renormalization ansatz, New J. Phys. 15, 023020 (2013).

[11] X. Peng, J. Du, and D. Suter, Quantum phase transition of ground-state entanglement in a Heisenberg spin chain simulated in an NMR quantum computer, Phys. Rev. A 71, 012307 (2005).
[12] X. Peng, Z. Luo, S. Kou, D. Suter, and J. Du, Experimental Implementation of Adiabatic Passage between Different Topological Orders, Phys. Rev. Lett. 113, 080404 (2014).

[13] E. H. Lieb and D. W. Robinson, The finite group velocity of quantum spin systems, Commun. Math. Phys. 28, 251 (1972).

[14] A. Bhattacharyya, L. Cheng, L. Y. Hung, S. Ning, and Z. Yang, Notes on the Causal Structure in a Tensor Network, arXiv:1805.03071.

[15] G. Vidal, Geometric tensor networks for critical quantum spin chains, KITP Conference: Frontiers of Quantum Information Physics (2017).

[16] M. Socolovsky, Rindler space, Unruh effect and Hawking, arXiv:1304.2833.

[17] J. J. Bisognano and E. H. Wichmann, On the duality condition for quantum fields, J. Math. Phys. (N.Y.) 17, 303 (1976).

[18] R. Haag, Local Quantum Physics: Fields, Particles, Algebras (Springer, Berlin, 1992).

[19] H. Casini and M. Huerta, Entanglement entropy in free quantum field theory, J. Phys. A 42, 504007 (2009).

[20] I. Peschel, On the reduced density matrix for a chain of free electrons, J. Stat. Mech. (2004) P06004.

[21] I. Peschel and V. Eisler, Reduced density matrices and entanglement entropy in free lattice models, J. Phys. A 42, 504003 (2009).

[22] K. Papadodimas and S. Raju, An infalling observer in AdS/CFT, J. High Energy Phys. 10 (2013) 212.

[23] A. Milsted and G. Vidal, Tensor networks as path integral geometry, arXiv:1807.02501.

[24] J. Cotler, X. Han, X. L. Qi, and Z. Yang, Quantum causal influence, arXiv:1811.05485. 